\title{
Cell-autonomous retinoic acid receptor signaling has stage-specific effects on mouse enteric nervous system
}

\author{
Tao Gao, ${ }^{1}$ Elizabeth C. Wright-Jin, ${ }^{2}$ Rajarshi Sengupta, ${ }^{1}$ Jessica B. Anderson, ${ }^{1}$ and Robert O. Heuckeroth ${ }^{1,2,3}$ \\ 'Children's Hospital of Philadelphia Research Institute, Philadelphia, Pennsylvania, USA. Department of Pediatrics, \\ Washington University School of Medicine, St. Louis, Missouri, USA. ${ }^{3}$ Department of Pediatrics, Perelman School of \\ Medicine at the University of Pennsylvania, Philadelphia, Pennsylvania, USA.
}

\begin{abstract}
Retinoic acid (RA) signaling is essential for enteric nervous system (ENS) development, since vitamin A deficiency or mutations in RA signaling profoundly reduce bowel colonization by ENS precursors. These RA effects could occur because of RA activity within the ENS lineage or via RA activity in other cell types. To define cell-autonomous roles for retinoid signaling within the ENS lineage at distinct developmental time points, we activated a potent floxed dominant-negative RA receptor $\alpha$ (Rar $\alpha \mathrm{DN}$ ) in the ENS using diverse CRE recombinase-expressing mouse lines. This strategy enabled us to block RA signaling at premigratory, migratory, and postmigratory stages for ENS precursors. We found that cell-autonomous loss of RA receptor (RAR) signaling dramatically affected ENS development. CRE activation of RaraDN expression at premigratory or migratory stages caused severe intestinal aganglionosis, but at later stages, RaraDN induced a broad range of phenotypes including hypoganglionosis, submucosal plexus loss, and abnormal neural differentiation. RNA sequencing highlighted distinct RA-regulated gene sets at different developmental stages. These studies show complicated context-dependent RA-mediated regulation of ENS development.
\end{abstract}

Conflict of interest: $\mathrm{ROH}$ is a consultant for BlueRock Therapeutics and served on a Scientific Advisory Board for Takeda.

Copyright: () 2021, Gao et al. This is an open access article published under the terms of the Creative Commons Attribution 4.0 International License.

Submitted: November 17, 2020

Accepted: April 7, 2021

Published: May 24, 2021

Reference information: /CI Insight. 2021;6(10):e145854.

https://doi.org/10.1172/jici.

insight.145854.

\section{Introduction}

The enteric nervous system (ENS) is a complex network of neurons and glia that resides in the bowel wall and is essential for intestinal function $(1,2)$. These ENS cells arise primarily from vagal enteric neural crestderived cell (ENCDC) precursors that divide rapidly and colonize the bowel in a rostral to caudal direction from E9 to E13.5 in mice (3-6). In addition to vagal ENCDC, ENS precursors include sacral neural crest (7), mesenteric neural crest (8), sympatho-enteric precursors (9), Schwann cells (10), and perhaps bowel epithelial cells (11). As ENCDC colonize the bowel, they differentiate into about 20 neuron types, and many types of glia that form extensive networks to control most aspects of bowel function $(2,12-17)$. Maturation of the ENS continues during fetal development, and remodeling continues after birth (18-20). Retinoic acid (RA), the active metabolite of vitamin $\mathrm{A}$, is an important morphogen with an integral role in ENCDC migration, proliferation, and differentiation (21-32). RA functions mainly as a transcriptional regulator that binds to and activates RA receptor/retinoid $x$ receptor (RAR/RXR) heterodimers (33). RAR/RXR heterodimers bind DNA at RA response elements (RAREs) to regulate transcriptional activity. Several studies demonstrate vital roles for vitamin A and its active metabolite RA in ENS development. In rat and mouse primary cell culture, RA increases ENCDC proliferation and neuronal differentiation while decreasing neurite length $(23,27)$. In vivo, vitamin A-deficient mice ( $R b p 4^{-1-}$ mice on a vitamin A-deficient diet) develop distal bowel aganglionosis (26), confirming that vitamin A is needed for bowel colonization by ENCDC. The extent of bowel aganglionosis dramatically increased when Ret heterozygosity was combined with vitamin A deficiency, suggesting potent gene-environment interactions in mice (26). Consistent with these observations, loss of the primary RA synthesis enzyme retinaldehyde dehydrogenase 2 (Raldh2) also causes severe intestinal aganglionosis in mice (29), with more minor effects of murine Raldh1 and Raldh3 mutations (25). Furthermore, maternal vitamin A deficiency or excess can cause intestinal hypoganglionosis without aganglionosis in rats and mice $(22,28,32)$. While these results clearly show that RA signaling is needed for ENS development, many questions remain. First, because RAR and RXR receptors are expressed in diverse cell types (27), it is not clear whether RA acts directly on ENCDC or via effects on neighboring cells. Second, effects of RA signaling at distinct developmental stages remain elusive. Third, the RA-targeted gene network in ENCDC in vivo is not yet established. To address these questions, we employed a very potent 
Table 1. RaraDN ${ }^{L O x P /+}$; Wnt1Cre ${ }^{+}$embryos obtained at various ages

\begin{tabular}{lccccccc}
\hline Age & $\begin{array}{c}\text { Litters } \\
\text { analyzed }\end{array}$ & $\begin{array}{c}\text { Total embryos } \\
\text { analyzed }\end{array}$ & $\begin{array}{c}\text { Expected RaraDN; } \\
\text { Wnt1Cre number }\end{array}$ & $\begin{array}{c}\text { Actual RaraDN; } \\
\text { Wnt1Cre number }\end{array}$ & $\chi^{2}$ statistic & $P$ value \\
\hline E10.5 & 5 & 41 & 10.25 & 10 & 0.004 & 0.949571 \\
\hline E11.5 & 14 & 109 & 27.25 & 37 & 2.098 & 0.147492 \\
E12.5 & 22 & 147 & 36.75 & 26 & 0 & 2.341 & 0.126009 \\
\hline E14.5 & 5 & 34 & 8.5 & & & & \\
\hline
\end{tabular}

CRE-dependent dominant-negative RA receptor $\alpha$ (Rar $\left.\alpha D^{\text {Loxp-STOP-LoxP/+} ; ~ R A R ~} \alpha \mathrm{DN}\right)(34)$ in combination with fluorescence-based lineage tracing in mice. We discovered that RA signaling regulates ENS development in a cell-autonomous manner, with distinct effects on different developmental stages. Furthermore, gene expression profiling showed stage-specific effects of blocking RAR signaling in developing ENCDC. These results suggest that vitamin A deficiency or excess could alter ENS structure and function in many ways during intrauterine and postnatal periods, contributing to human bowel motility disorders.

\section{Results}

Cell-autonomous RAR signaling in neural crest derivatives is required for craniofacial and ocular development. To characterize cell-autonomous roles for RAR signaling within the ENS lineage, we bred RaraDN ${ }^{\mathrm{LoxP} /+}$ to $\mathrm{Wnt}_{1 \mathrm{Cre}}{ }^{+}$ mice. $R a r \alpha D N^{L o x P /+}$ produce RAR $\alpha \mathrm{DN}$ after CRE-mediated DNA recombination. Wnt1Cre express Cre recombinase in the CNS and many neural crest derivatives, including essentially all fetal ENS precursors (35-37).

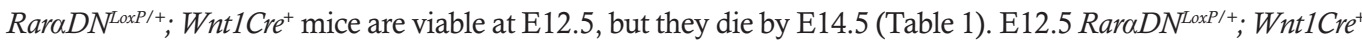
have major malformations of neural crest-derived facial structures (Figure 1, A and B) with absent facial cartilage (Figure 1, C and D). Sectioning showed failure of nasomedial process fusion at the midline and a wide frontonasal region (Figure 1, E and F). In contrast, dorsal root ganglia (DRG), another crest-derived structure, appeared fairly normal even though Wnt1Cre induced recombination of an EYFP reporter in DRG (Figure 1, G-L). These data highlight distinct RA roles in different neural crest-derived tissues during development. Our primary goal was to investigate RA signaling effects in the ENS.

ENS development requires cell autonomous RAR signaling. At E12.5 in Wnt1Cre (control) mice, ENCDC had colonized the esophagus, stomach, small intestine, and half of the colon, as seen by TuJ1 (neuron specific $\beta 3$-tu-

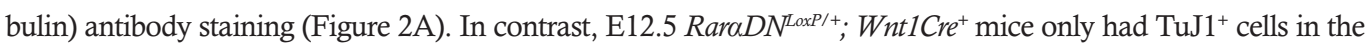
esophagus and stomach (Figure $2 \mathrm{~B}$ ). Recognizing that the absence of TuJ1 staining might reflect impaired neuronal differentiation in RaraDN ${ }^{L o x P /+} ;$ Wnt $1 C r e^{+}$mice or could reflect absent ENS, we bred to R26R-TdTomato lineage reporter mice so that cells undergoing CRE-mediated DNA recombination are unambiguously marked. While TdTomato $^{+}$ENS and $\mathrm{HuC} / \mathrm{D}^{+}$(neuronal RNA binding protein) neurons were readily detected in stomach, small bowel, and proximal colon of control animals (Figure 2, C-E and I), the RaraDN ${ }^{\text {LoxP/+}}$; Wnt1Cre ${ }^{+}$R26R-TdToma$t^{+}$mice did not have TdTomato ${ }^{+}$or $\mathrm{HuC} / \mathrm{D}^{+}$cells in the small bowel or colon and had fewer ENS cells in stomach than controls (Figure 2, F-H and J). These analyses confirmed that blocking cell-autonomous RAR signaling in the Wnt $1 \mathrm{Cre}$ lineage completely prevented these ENS precursors from colonizing the small bowel and colon.

Blocking RAR signaling causes defects in ENCDC migration and differentiation by E10.5. To determine if RaraDN expression within neural crest-derived ENS precursors acts at earlier developmental stages, we examined E10.5 whole embryo via 3DISCO tissue clearing (38) and confocal microscopy (Figure 3, A and B). TuJ1 and SOX10 antibody staining showed many ENCDC in the esophagus and stomach in control mice (Figure 3, C-F) but very few stained ENCDC in the proximal bowel of RaraDN ${ }^{L o x P /+}$; Wnt1Cre ${ }^{+}$mice (Figure 3, G-J), consistent with a defect in early stages of bowel colonization. More specifically, while there were some SOX $10^{+} \mathrm{ENCDC}$ near the vagus in mutant mice (Figure 3, G-J), the control mice had many SOX10+ ENCDC that had migrated far beyond the vagus and into the stomach (Figure 3, C-F). Furthermore, in control mice, many ENCDC were TuJ1 immunoreactive, suggesting early neuronal differentiation (Figure 3, C and E), but there were almost no TuJ1 ${ }^{+}$cells in the esophagus or stomach of $\mathrm{RaraDN}^{\mathrm{LoxP} /+}$; Wnt1Cre ${ }^{+}$mice (Figure 3, G and I). Collectively, these data suggest that cell-autonomous RAR signaling is needed for ENCDC to populate the bowel and differentiate into neurons.

Cell autonomous RAR signaling is required for RET and PHOX2B expression in ENCDC. The defect in bowel colonization by ENCDC of RaraDN $N^{L o x P /+} ; W n t 1 C r e^{+}$mice closely resembles the phenotype in Ret and Phox $2 b$ null mice 

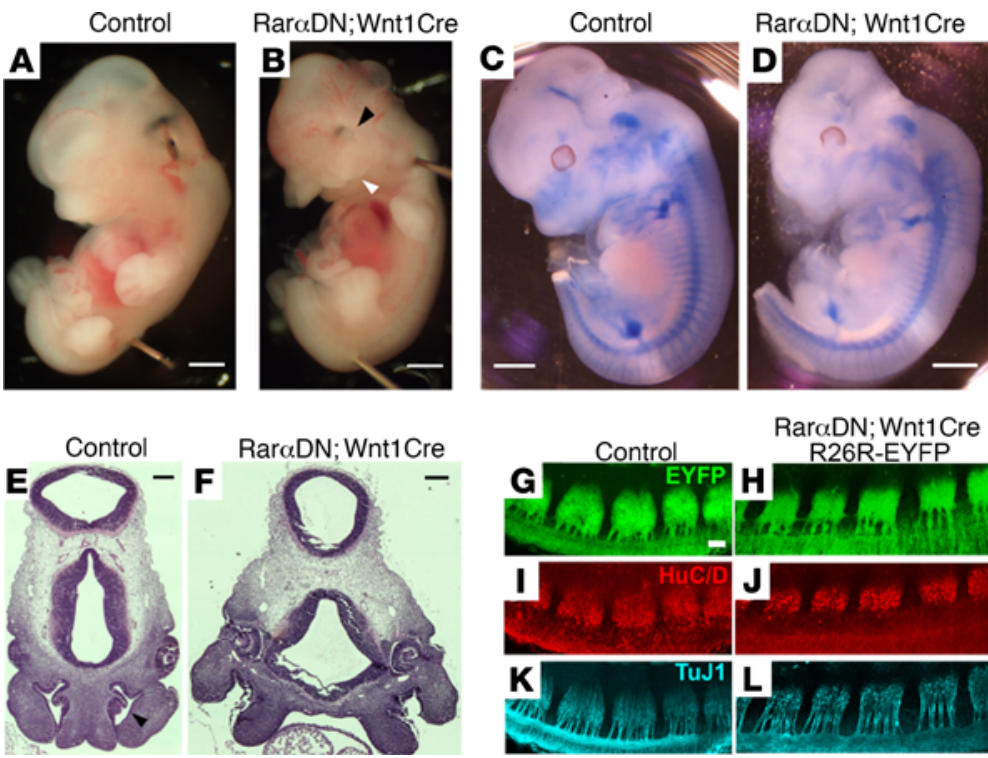

RaraDN; Wnt1Cre

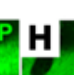
R26R-EYFP

Figure 1. RaraDN ${ }^{\text {LoxP/++} ; ~ W n t 1 C r e+~ m i c e ~ h a v e ~ a b n o r m a l ~ c r a n i o f a c i a l ~ d e v e l o p m e n t . ~(A ~ a n d ~ B) ~ E 12.5 ~ R a r a D N ~} N^{L o x P /+;}$ Wnt1Cre (RaraDN; Wnt1Cre) mice have obvious craniofacial defects with inset eyes (black arrowhead) and midline facial cleft (white arrowhead). Scale bar: $1 \mathrm{~mm} . n=3$ control, $n=3$ RaraDN $N^{\text {LoxP/+} ; ~ W n t 1 C r e+. ~(C ~ a n d ~ D) ~ E 12.5 ~ A l c i a n ~ b l u e ~ s t a i n i n g ~ s h o w s ~}$ markedly reduced cartilage in neural crest-derived structures of the face. Scale bar: $1 \mathrm{~mm} . n=3$ control, $n=3$ Rar $\alpha D N^{L \times x P /+}$; Wnt1Cre+. (E and F) Representative images of H\&E-stained coronal sections of RaraDN ${ }^{L a x P /+}$; Wnt1Cre ${ }^{+}$mice show inset eyes and cleft nasal passages compared with control $($ Wnt1Cre+) at E12.5. Arrowhead indicates nasal cavity. Scale bar:

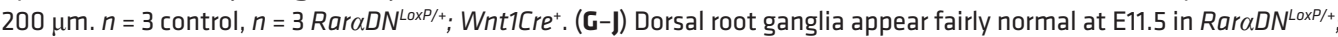

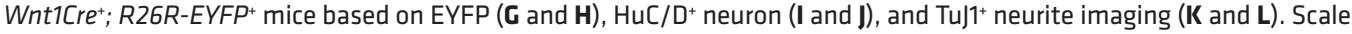
bar: $100 \mu \mathrm{m} . n=3$ control, $n=3$ Rar $\alpha D N^{L \alpha x P /+} ; W_{n t 1 C r e}$.

$(39,40)$ (Figure 4, A-C). Because RA was previously reported to induce Ret expression in quail ENCDC migrating from the neural tube to bowel (24), we stained E12.5 bowel from RaraDN ${ }^{L o x P /+} ; W n t 1 C r e^{+} ; R 26 R-E Y F P^{+}$and controls ( Wnt 1 Cre ${ }^{+} ;$R26R-EYFP ${ }^{+}$) with RET antibody. A total of $100 \%$ of stomach $\mathrm{EYFP}^{+} \mathrm{ENCDC}$ expressed RET in controls as expected (Figure 4, D-F). Curiously, while $1 \mathrm{RaraDN}^{\mathrm{LoxP} /+} ; \mathrm{Wnt}_{1 C r e^{+}} ; \mathrm{EYFP}^{+}$mouse had many $\mathrm{RET}^{+} \mathrm{EYFP}^{+} \mathrm{ENCDC}$ in the stomach (42\%), the other mice evaluated had very few $\mathrm{RET}^{+} \mathrm{EYFP}^{+} \mathrm{ENCDC}(0 \%$, $0 \%, 0.4 \%$, and $4.9 \%$ ) (Figure $4, \mathrm{G}-\mathrm{J}$ ). $\mathrm{RAR} \alpha \mathrm{DN}$ also dramatically reduced the percentage of lineage-marked ENCDC that express PHOX2B in the stomach or esophagus of $\mathrm{RaraDN}^{\mathrm{LoxP} /+}$; Wnt1Cre ${ }^{+}$; TdTomato mice (Figure 5, A-F and H). EdU incorporation into lineage marked ENCDC in E12.5 stomach of RaraDN ${ }^{L o x P /+}$; Wnt $1 \mathrm{Cre}^{+}$, TdTomato mice, however, was not statistically different from controls (Figure 5, A-F and G); therefore, it is not clear that reduced proliferation within stomach ENCDC fully accounts for the phenotype. Collectively, these data suggest that RAR signaling is needed in the Wnt1Cre ENS lineage for Ret and Phox $2 b$ expression, further suggesting that loss of either RET or PHOX2B could cause this type of extensive intestinal aganglionosis $(39,40)$

Loss of RAR signaling causes defective vagal nerve development. In parallel with the loss of ENCDC in fetal stomach, E11.5 RaraDN ${ }^{\mathrm{LoxP} /+}$; Wnt1Cre+ mice had smaller vagus nerves than control littermates (Figure 6, A-C). The reduction in vagal nerve fibers in the stomach appears to be non-cell autonomous because $\mathrm{TuJ} 1^{+}$vagal nerve fibers are TdTomato-negative in $\mathrm{Wnt}_{1 \mathrm{Cre}}$; TdTomato $^{+}$and $\mathrm{RaraDN}^{+}$; Wnt $1 \mathrm{Cre}^{+}$; TdTomato $^{+}$mice (Figure $6 \mathrm{D}$, Supplemental Figure 1, and Supplemental Videos 1 and 2; supplemental material available online with this article; https://doi.org/10.1172/jci.insight.145854DS1). Enlarged images also demonstrate many TdTomato ${ }^{+}$ cells migrating along the TuJ1+ vagal nerve fibers in $\mathrm{Wnt}_{1} \mathrm{Cre}^{+} ; \mathrm{TdTomato}$ and $\mathrm{Rar \alpha DN} ; \mathrm{Wnt} 1 \mathrm{Cre}^{+}$; $\mathrm{TdTomato} \mathrm{mice}^{+}$ (Supplemental Figure 1, E, F, K, and L). Furthermore, while ENCDC migrate far beyond the vagus in control animals, TuJ1+- and SOX10-labeled ENCDC remained close to vagal fibers in E11.5 RaraDN ${ }^{\text {LoxP/+ }}$; Wnt $1 \mathrm{Cre}^{+}$ mice (Figure 3, D and H, and Figure 6, A and B). These observations suggest synergistic interactions between growing vagal nerve fibers and migrating ENCDC that colonize the bowel to form ENS.

Loss of RAR signaling in SOX10 lineage of ENCDC causes aganglionosis. To evaluate cell-autonomous effects of RAR beyond E12.5 when RaraDN ${ }^{\mathrm{LoxP} /+} ;$ Wnt1Cre $e^{+}$die, we generated RaraDN ${ }^{\mathrm{LoxP} /+}$; SOX10Cre mice. These mice express Crefrom Sox10 regulatory elements, beginning at E8.5 when ENCDC migrate through the paraxial mesoderm to foregut (41). To confirm CRE activation in the ENS lineage, we examined SOX10Cre; R26R-TdTomato 

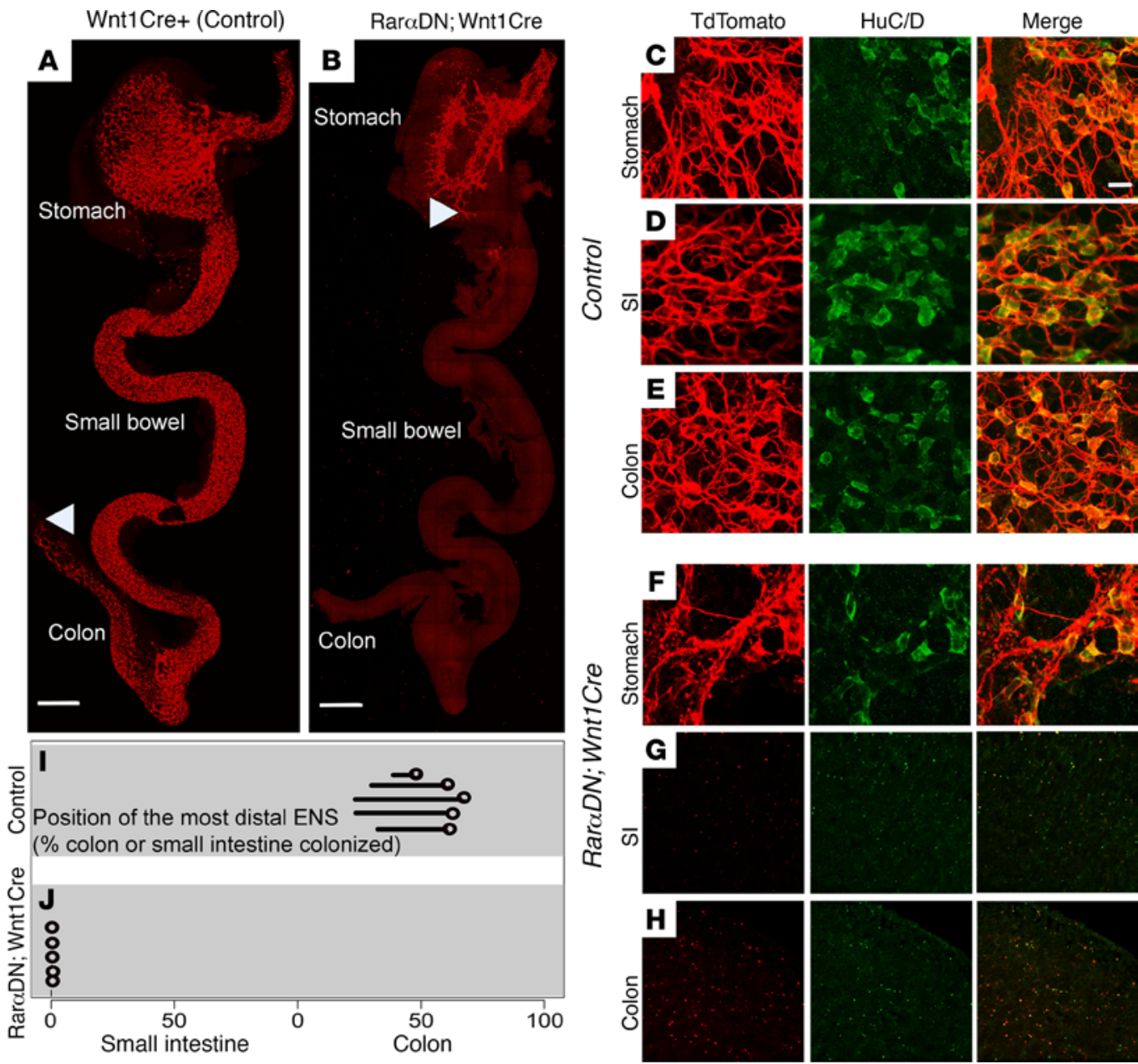

Figure 2. Cell-autonomous RAR signaling is required for ENS precursor colonization of fetal small bowel and colon. (A and B) Representative images of bowel colonization by Tul1+ enteric neurons (red) at E12.5. (A) Wnt1Cre controls

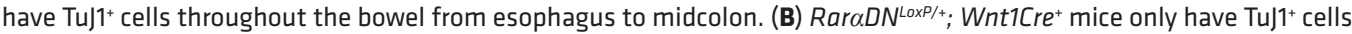
present in the esophagus and stomach. A white arrowhead shows the position of the most distal Tuj1 $1^{+}$cell or neurite in each image. Scale bars: $500 \mu \mathrm{m}$. (C-H) Representative E12.5 images of stomach, small intestine (SI), and colon show cells stained with $\mathrm{TdTomato}^{+}$(red) and HuC/D (green) throughout the bowel in control (C-E), whereas TdTomato ${ }^{+}$and HuC/D-stained cells are absent in SI and colon of RaraDN $N^{\text {LoxP/+ }}$; Wnt1Cre+ mice (F-H). (C-H) Scale bar: $150 \mu \mathrm{m}$. (I and J) Circles show the position of the most distal TdTomato+ ENS cell in control or RaraDN ${ }^{\text {LoxP/+ }}$; Wnt1Cre mice at E12.5. The line attached to each circle indicates a hypoganglionic zone in controls. $n=5$ controls, $n=5$ RaraDN ${ }^{L a x P /+} ; W_{n t 1 C r e^{+}}$

mice at E12.5 and found essentially complete overlap of the TdTomato lineage marker and RET antibody staining in the bowel (Supplemental Figure 2). Unlike controls (Figure 7A) and like RaraDN ${ }^{\mathrm{LoxP} /+}$; Wnt1Cre ${ }^{+}$, the E12.5 RaraDN ${ }^{\text {LoxP/+}}$; SOX10Cre $e^{+}$had TuJ1 $1^{+}$ENCDC predominantly confined to the stomach at E12.5 (Figure 7, B-E), with a few sparsely distributed TuJ1+ ENCDC in the proximal small intestine (Figure 7D). By E15.5, Rar $\alpha D N^{\text {LoxP/+ }}$; SOX10Cre had obvious eye and craniofacial defects but remained viable (Figure 7F). The E15.5 ENS network was dense in the esophagus and stomach but was sparse in the proximal small intestine (Figure 7, G, J, L, and N) compared with controls (Figure 7, I, K, and M). Distal small intestine (Figure 7P) and colon were aganglionic with extrinsic nerve fibers in the distal colon (Figure 7, $\mathrm{H}$ and $\mathrm{R}$ ) of mutant mice in contrast to controls (Figure 7, O and Q). Neurons in the small bowel were often associated with extrinsic nerve fibers and separated from more proximal ENS (Figure 7, S-V). These data are consistent with the hypothesis that RAR signaling is required as ENCDC migrate from the neural tube to bowel and that it is not needed by ENS precursors before $\mathrm{E} 8.5$ in the neural tube.

Blocking RAR signaling in the TyrCre lineage reduces enteric neurons and alters ENS patterning. We next examined the ENS in mice that express Cre in ENCDC from the tyrosinase promoter (RaraDN $\mathrm{LxPP}^{\mathrm{L} /+} ; \mathrm{Tyr} C r e^{+}$) starting at E10.5 $(41,42)$ when ENCDC normally reach the midgut (3). In these mice, the extent of bowel colonization by 

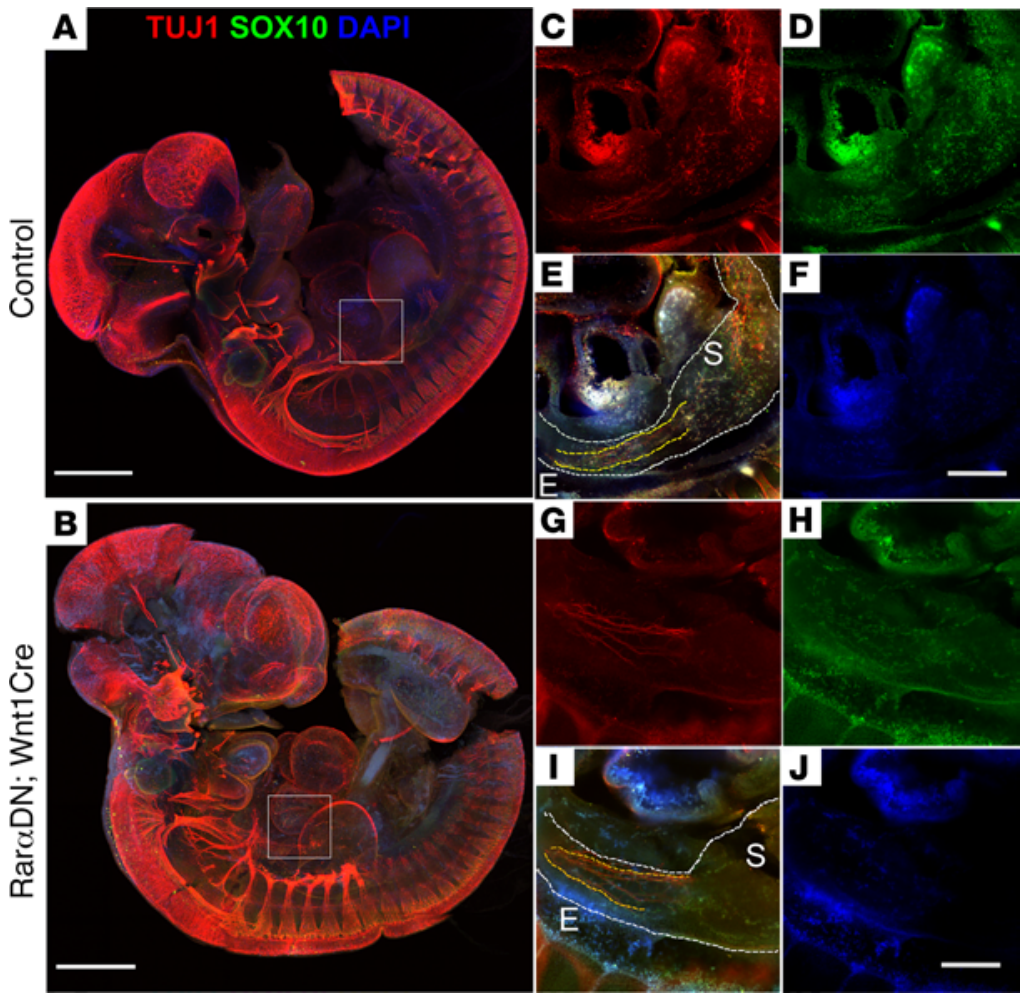

Figure 3. Blocking RAR signaling impairs ENCDC migration and differentiation at E10.5. (A and B) Whole embryo imaging showing E10.5 RaraDN LoxP/+ (control) (A) or E10.5 RaraDN LoxP/+; Wnt1Cre+ ${ }^{+}$(B) mice stained with TUJ1 (red) and SOX10 (green) antibodies plus DAPI (blue). Scale bar: $500 \mu \mathrm{m}$. (C-J) Zoomed-in images of selected slices from the Z-stack of the whole

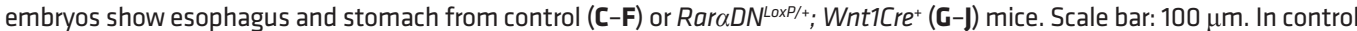
embryos, there are readily visible SOX10+TU $11^{+}$cells in both esophagus and stomach area (C-F). In contrast, there are almost no SOX10+ cells in the mutant stomach, even though SOX10+ cells were observed in the esophagus (G-J). (E and I) White dotted lines outline of esophagus and stomach. Yellow dotted lines highlight the region with extrinsic vagal nerve fibers. E, esophagus; S, stomach. These are representative images from $n=3$ Rar $\alpha \mathrm{DN}^{\mathrm{LoxP} /+}$ (control), $n=3$ RaraDN $\mathrm{NoxP/+}^{\mathrm{L}}$ Wnt1Cre+ .

ENCDC was similar to controls at E13.5 (Figure 8, A and E). Stomach ENS appeared normal in $\operatorname{RaraDN}^{\operatorname{LoxP} /+}$, $\mathrm{TyrCre}^{+}$mice (Figure 8, B and $\mathrm{F}$ ), but the small bowel and colon were hypoganglionic relative to controls (Figure 8, C, D, G, and H). ENS organization was also abnormal in the small bowel and colon of mutants at E13.5. Instead of the fine network of cells seen in controls, RaraDN ${ }^{\text {LoxP } /+} ;$ TyrCre $^{+}$ENS had thick chains of cells (Figure 8, A, C-E, G, and H). At birth, RaraDN LoxP/+; TyrCre+ mice appeared healthy but did not feed (absent milk spot, Figure $8 \mathrm{I})$ and consistently died within 12 hours $(n=6)$. To avoid analysis of dying mice, we examined E18.5 ENS. Although the RaraDN ${ }^{\text {LoxP/+ }} ;$ TyrCre $^{+} ;$R26R-TdTomato bowel looked grossly normal (Figure 8J), lineage-marked TdTomato ${ }^{+}$ENS cells were less dense in the mutant bowel throughout the small intestine and colon (Figure 8K). HuC/D IHC confirmed that enteric neurons were sparse (Figure 8, L, and N-P) and that all neurons in RaraDN LoxP $/+^{+}$TyrCre ${ }^{+}$; R26R-TdTomato bowel were TdTomato ${ }^{+}$(Figure 8, L and M). To determine if blocking RAR impacted neuron subtype ratios, we counted NOS1 and HuC/D double-labeled cells and discovered that more $\mathrm{HuC} / \mathrm{D}^{+}$neurons were $\mathrm{NOS}^{+}$in $\mathrm{RaraDN}^{\mathrm{LoxP} /+}$; TyrCre ${ }^{+}$ENS (Figure 8, Q-S) compared with controls. Collectively, these results show that cell-autonomous RAR signaling is important after E10.5 for ENS patterning, to increase neuron number, and for neuron subtype specification.

Inactivation of RAR signaling in the RET lineage causes distal bowel hypoganglionosis. Sparse ENS in the preceding models might reflect inadequate RET, making it difficult to identify other roles for RAR signaling in ENCDC. Our prior studies suggest that RA is not needed to sustain Ret expression in ENCDC that already express Ret $(26,27)$. We therefore decided to use mice expressing CRE-ERT2 from the Ret locus to activate Rar $\alpha D N$ expression in ENCDC that already express Ret. For these experiments, we also needed a CRE-dependent fluorescent reporter (EYFP) to track CRE activity. Because Ret is on mouse chromosome 6 near the ROSA26 locus that drives expression of RaraDN, as well as most fluorescent reporters, we bred $\operatorname{Rar} \alpha D N^{L o x P /+}$ mice to $R E T$ CreERT2-EYFP Tandem mice to generate RaraDN ${ }^{\mathrm{LoxP} /+} ;$ RETCreERT2-EYFP Tandem and RaraDN $N^{\mathrm{LLxP} /+}$ controls. The 

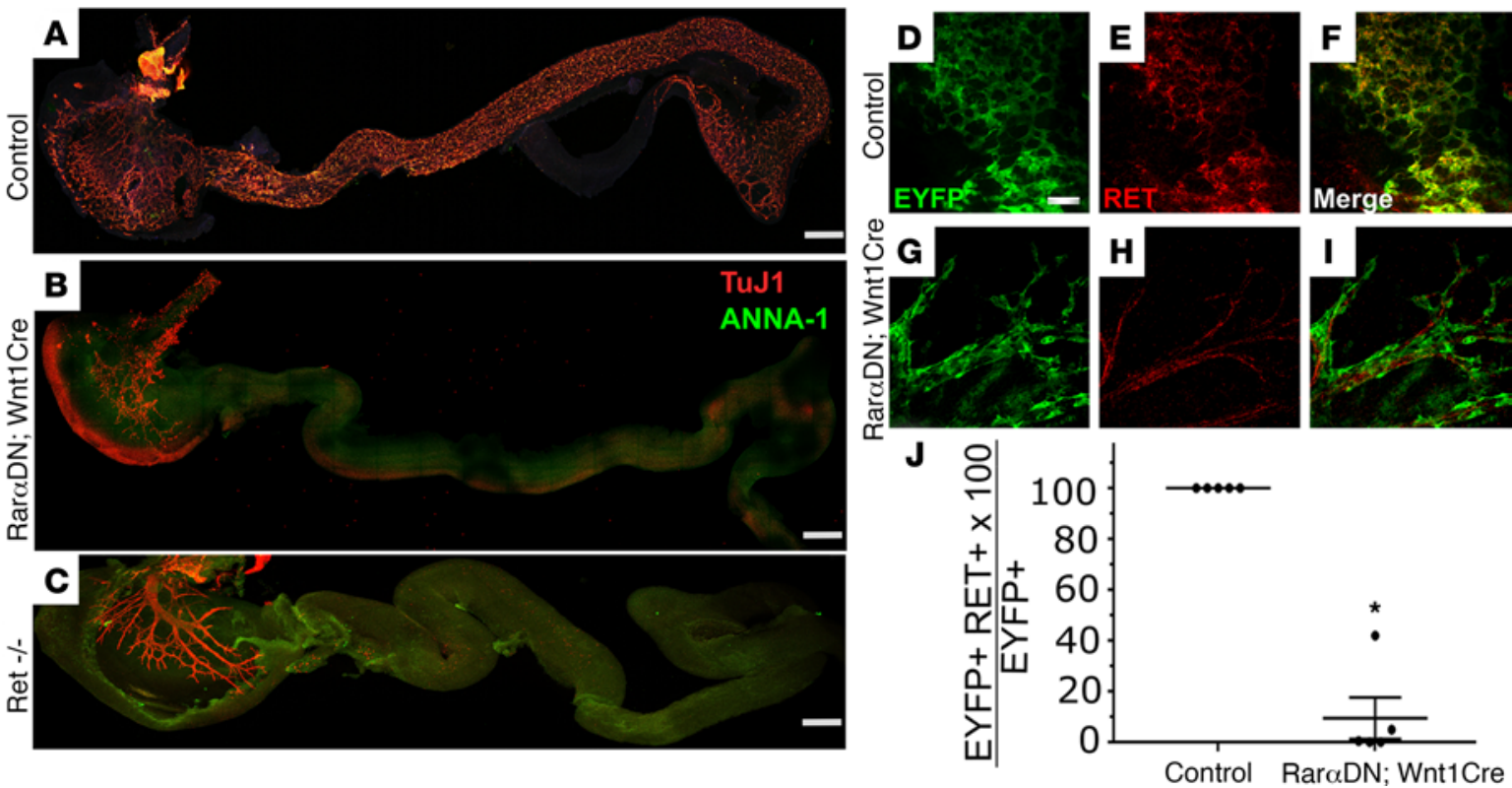

Figure 4. RAR signaling in ENCDC is required for Ret expression. (A-C) Whole-mount TuJ1 and HuC/D antibody staining of E12.5 bowel from control,

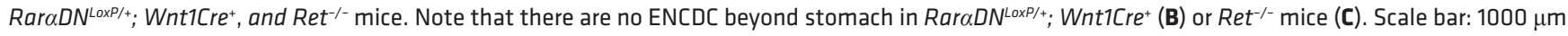
(D-I) IHC of E12.5 control (Wnt1Cre+; Rosa26 EYFP) (D, E, and F) or RaraDN $N^{\text {LoxP/+ }}$; Wnt1Cre ${ }^{+}$; Rosa26 EYFP (G, H, and I) stomach using EYFP and RET antibodies. Scale bar: $50 \mu \mathrm{m}$. (J) Percentage ratio of EYFP+ ENCDC that are RET immunoreactive. Wnt1Cre+; Rosa26EYFP ( $n=5$ mice, 2100 cells), RaraDN LoxP/+; Wnt1Cre+; Rosa26 EYFP ( $n=5$ mice, 1994 cells, $P=0.0002$, 2-tailed unpaired Student's $t$ test).

RaraDN $N^{\text {LoxP/+}}$; RETCreERT2-EYFP Tandem have undergone recombination so RETCreERT2 and a CRE-activated EYFP-Channelrhodopsin-2 (ChR2) protein are on the same chromosome. E13.5 control RaraDN ${ }^{\text {LoxP } /+}$ lacking CreERT2 had the bowel fully colonized by ENS precursors after E10.5 tamoxifen treatment (Figure 9, A, B, D, F, and H). E10.5 tamoxifen-treated RaraDN ${ }^{\text {LoxP/+ }}$; RETCreERT2-EYFPTandem also had normally patterned ENS in the esophagus and small bowel (Figure 9, A, C, E, and G) but had hypoganglionic ENS in the colon, with thick chains of ENS cells (Figure 9,C and I). We confirmed high levels of CRE-activation in the ENS after tamoxifen treatment and almost no CRE activation in the absence of tamoxifen in these RETCreERT2-EYFPTandem mice (Supplemental Figure 3). Because tamoxifen effects may take 12-18 hours, we also tamoxifen treated at E8.5 but found similar phenotypes (Figure 9, J-M). RET and PHOX2B were readily detected in $\mathrm{EYFP}^{+} \mathrm{ENCDC}$ of E13.5 RaraDN ${ }^{\mathrm{LoxP} /+}$; RETCreERT2-EYFP Tandem mice, consistent with our prior studies (26, 27). These data suggest that RAR is needed to activate RET and PHOX2B expression in ENCDC (Figure 4, D-I, and Figure 5, A-F and $\mathrm{H}$ ) but not to maintain expression once regulatory elements are activated (Figure 9, N-Q). The unusual patterning in the distal colon of tamoxifen-treated E13.5 RaraDN LoxP/+ $^{2}$ RETCreERT2-EYFPTandem mice suggests that RAR regulates additional ENS patterning genes in ENCDC.

Ret lineage loss of RAR signaling profoundly reduced submucosal neuron density and altered cell identity. Rar $\alpha N^{\text {LoxP/+ }}$; RETCreERT2-EYFP Tandem treated with tamoxifen at E10.5 (Figure 10) were born at a normal Mendelian ratio, grew normally, and had a normal-appearing bowel (Figure 10B), permitting analysis of adult ENS. We therefore stained the bowel of 2-month-old mice with $\mathrm{HuC} / \mathrm{D}$ and TuJ1 antibodies (Figure $10, \mathrm{C}-\mathrm{N})$. Quantitative analysis demonstrated a $50 \%-65 \%$ reduction in myenteric neurons in small bowel and colon and a $90 \%$ loss of submucosal neurons in RaraDN-expressing mutant mice (Figure 10, O-T). In contrast to neurons, adult enteric glia marked by SOX10 antibody appeared similar in abundance in tamoxifen-treated RaraDN ${ }^{L o x P /+} ;$ RETCreERT2-EYFP Tandem and control mice (Supplemental Figure 4A). Interestingly, E10.5 tamoxifen treatment also led to a dramatic increase in $\mathrm{NOS}^{+}$submucosal neurons in $\operatorname{Rar} \alpha D N^{\operatorname{LoxP} /+}$; RETCreERT2-EYFP Tandem small bowel (Supplemental Figure 4B), and a mild increase in NOS1 ${ }^{+}$myenteric neurons. These studies confirm distinct age-dependent effects of cell-autonomous RAR signaling in the ENS.

Transcriptional profiling shows that RAR $\alpha$ N differentially impacts gene expression in E11.5 stomach and E13.5 colon $E N C D C$. Because RAR regulates transcription, we performed pooled-cell RNA sequencing (RNA-seq) to characterize RAR $\alpha \mathrm{DN}$-induced changes in ENCDC gene expression. For RaraDN ${ }^{\mathrm{LoxP} /+} ; \mathrm{Wnt}_{1} \mathrm{Cre}^{+} ; \mathrm{R}_{26 \mathrm{R}-\mathrm{TdTo}}$ mato mice, we analyzed E11.5 stomach ENCDC because RAR $\alpha$ DN eliminated ENCDC beyond the stomach. 

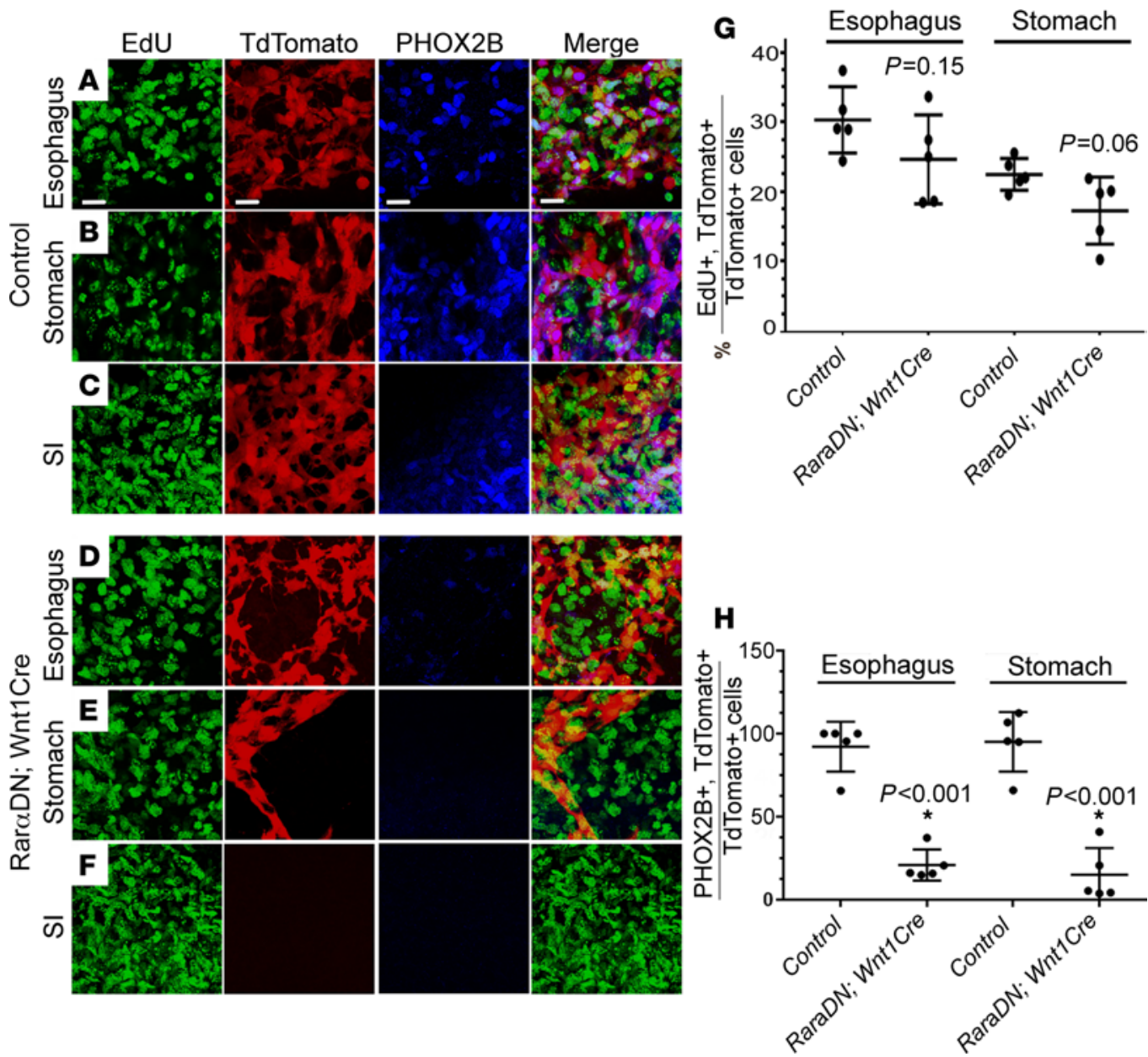

Figure 5. RAR signaling in ENCDC is required for PHOX2B expression. (A-F) Wnt1Cre+; R26R-TdTomato (control) and RaraDN ${ }^{\text {LoxP/+} ; ~ W n t 1 C r e+; ~ R 26 R-T d T o m a t o ~ E 12.5 ~ b o w e l ~ w h o l e-m o u n t ~ s h o w i n g ~ E d U ~ a n d ~ P H O X 2 B ~ I H C, ~ a s ~ w e l l ~ a s ~ T d T o m a-~}$ to fluorescence in esophagus ( $\mathbf{A}$ and $\mathbf{D}$ ), stomach (B and $\mathbf{E}$ ), and small intestines (C and $\mathbf{F}$ ). (G) Cell proliferation rate (EdU ${ }^{+}$TdTomato $^{+} /$TdTomato $\left.^{+} \times 100\right)(n=5$ mice, 718 cells) was similar in Wnt1Cre $;$ R26R-TdTomato (control) and Rar $\alpha D$ $\mathrm{N}_{\text {LoxP/+ }}$ Wnt1Cre+ mice; R26R-TdTomato ( $n=5$ mice, 643 cells). (H) Quantitative analysis of PHOX2B expression in ENCDC (PHOX2B+TdTomato $/$ TdTomato $^{+} \times 100$ ) showed few mutant ENCDC are PHOX2B'. Wnt1Cre+; R26R-TdTomato (control) ( $n=5$ mice, 718 cells) and Rar $\alpha \mathrm{DN}^{\text {LoxP/+ }}$; Wnt1Cre $;$ R26R-TdTomato ( $n=5$ mice, 643 cells). Scale bar: $20 \mu \mathrm{m}$. Two-tailed unpaired Student's $t$ tests were used for statistics.

For RaraDN ${ }_{\text {LoxP/+}}$; RETCreERT2-EYFP Tandem, we analyzed E13.5 colon ENCDC after tamoxifen treatment at E10.5 because RAR $\alpha$ DN-expressing colon ENCDC had patterning defects. Controls expressed fluorescent protein in ENCDC but lacked RaraDN. Flow sorting cleanly separated fluorescent cells from other bowel cell types (Figure 11, A and B). Multidimensional scaling (MDS) showed separate groups of control versus RaraDN-expressing ENCDC (Figure 11, C and D). Among approximately 23,000 transcripts at each age, we found 1413 genes differentially expressed between control and RaraDN ${ }^{L o x P}{ }^{P+} ;$ Wnt $1 C r e^{+}$ENCDC $(\mathrm{FDR}<0.05)$ at E11.5 (Supplemental Table 1) and 1140 genes differentially expressed between control and $\operatorname{Rar}_{\mathrm{O}} \mathrm{DN}{ }^{\mathrm{LoxP} /+}$; RETCreERT2-EYFPTandem (FDR < 0.05) at E 13.5 (Supplemental Table 2), as represented in volcano plots (Figure 11, E and F). The top 20 upregulated and downregulated genes at E11.5 (Supplemental Figure 5A) and E13.5 (Supplemental Figure 5B) and main dysregulated pathways (Figure 12A for E11.5 and Figure 12B for E13.5) suggest that many distinct biological processes are influenced by RAR signaling.

One initially surprising feature at E13.5 was that many hemoglobin genes were less abundant in flow-sorted cells from RaraDN; RETCreERT2-EYFP Tandem compared with controls. We suspect that this occurred because Ret is expressed in the hematopoietic stem cell (HSC) lineage, where RAR supports erythropoiesis $(43,44)$ and hemoglobin genes are normally expressed at high levels. Consistent with this hypothesis, some RETCreERT2-EYFP Tandem-lineage cells in the colon were stained with TER119 (erythroid lineage) and CD31 (endothelial, platelet, and leucocyte lineage) antibodies (Supplemental Figure 6). To define a gene set clearly linked to the ENS, we 

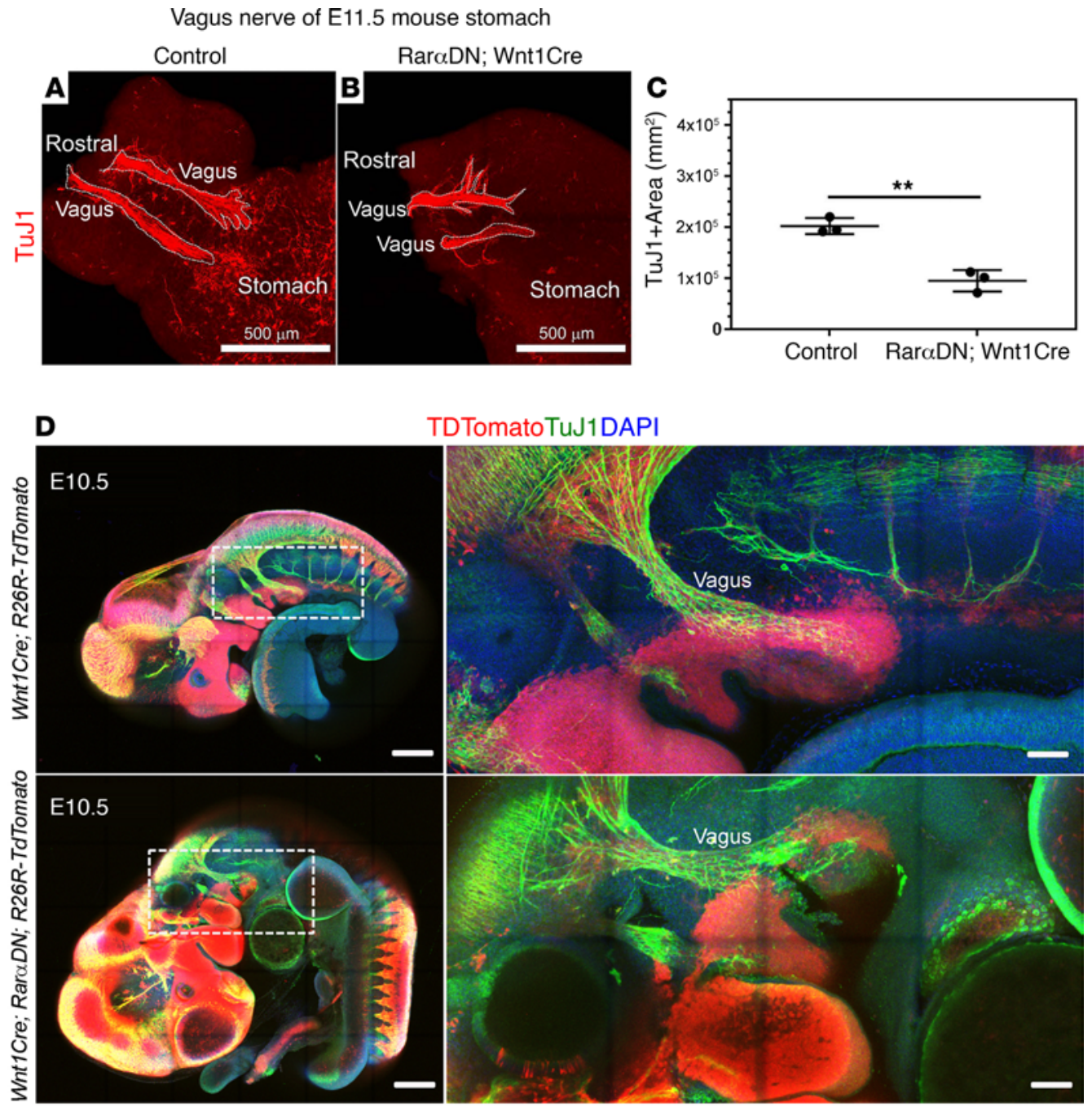

Figure 6. Vagal nerve fibers occupy a smaller area in E11.5 stomach of RaraDN ${ }^{\text {LoxP/+ }}$; Wnt1Cre ${ }^{+}$mice than in controls. (A and B) TuJ1 antibody-stained E11.5 stomach of RaraDN LoxP/+ (control) (A) or RaraDN LoxP/+; Wnt1Cre+ (B) mice. Scale bar: $500 \mu \mathrm{m}$. (C) Quantification of Tuj1+ stained vagal fiber area. ${ }^{* *} P<0.01$ by 2 tailed unpaired Student's $t$ tests. (D) Whole

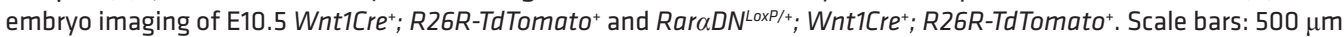
(left) and $200 \mu \mathrm{m}$ (right, enlarged). Note that Tuj1+ vagal nerve fibers are not TdTomato ${ }^{+}$. Box shows region of the magnified image. $n=3$, Wnt1Cre+; R26R-TdTomato ${ }^{+} . n=3$, RaraDN ${ }^{\text {LoxP/++} ; ~ W n t 1 C r e+; ~ R 26 R-T d T o m a t o+~}$. Supplemental Video 1 and Supplemental Video 2 show 3 -dimensional Z-stacks from embryos in $\mathbf{D}$.

compared differentially expressed genes in RaraDN; RETCreERT2-EYFPTandem and RaraDN; Wnt1Cre; TdTomato mice. In total, 594 genes were differentially regulated versus age-matched control cells in both mouse lines (Supplemental Figure 7 and Supplemental Table 3). Of these, a core set of 115 genes were regulated in the same direction by RAR $\alpha$ DN in ENCDC at E11.5 and E13.5 (Supplemental Tables 4 and 5). Gene enrichment pathway analysis of the aforementioned 2 sets of genes showed many pathways related to neuron development (Supplemental Figure 7). To validate RNA-seq data, we selected 2 differentially expressed genes of ENCDC at E11.5 (Stmn2 and Pax3) and performed quantitative PCR (qPCR), which confirmed differences predicted by RNA-seq (Supplemental Figure 8). The RNA-seq data for Ret and Phox2B in E11.5 stomach also correlate well with our IHC for RET and PHOX2B protein (Figure 4, Figure 5, and Supplemental Figure 9).

\section{Discussion}

RA regulates activity of the RAR/RXR transcription factor family to alter gene expression and influence many aspects of development (33). Prior studies using constitutive KO mice, RAR antagonist, stem cells, or vitamin A depletion in mice and rats show critical roles for RA in the developing ENS (21-32). For example, loss of the RA biosynthetic enzyme RALDH2 causes extensive bowel aganglionosis in Raldh2 ${ }^{-/-}$mice 

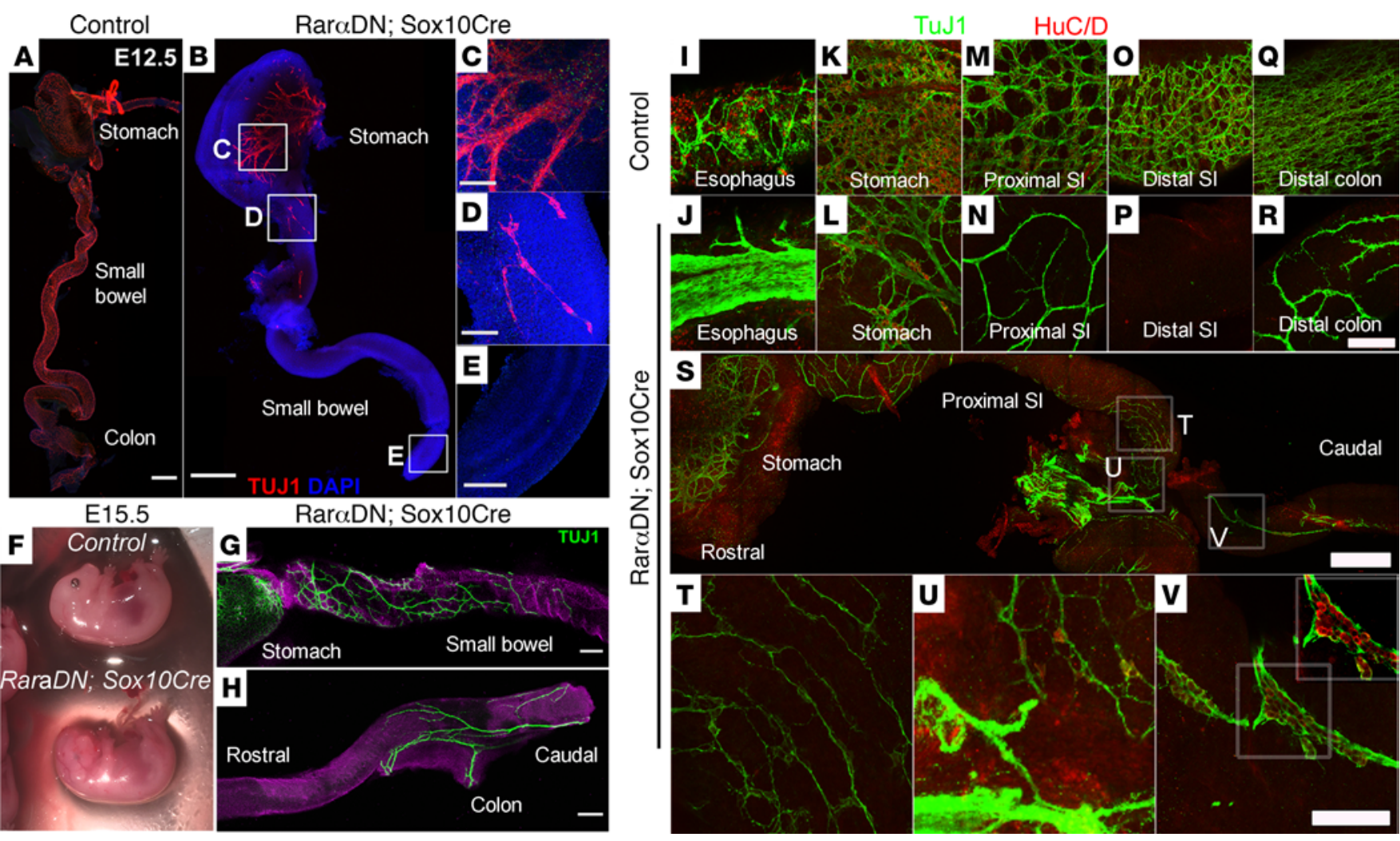

Figure 7. RaraDN ${ }^{\text {LoxP/++} ; ~ S o x 10 C r e+~ m i c e ~ h a d ~ e x t e n s i v e ~ d i s t a l ~ b o w e l ~ a g a n g l i o n o s i s . ~(A-E) ~ E 12.5 ~ c o n t r o l ~ o r ~ R a r ~} \alpha \mathrm{DN}^{\mathrm{LoxP} /+}$; Sox10Cre+ whole bowel was stained with TuJ1 antibody (red) and DAPI (blue). Insets highlight mutant stomach (C), proximal small intestine (D), and distal small intestine (E). Sparse TuJ1+ nerve cell bodies were seen in the proximal small bowel (D) but not in more distal small bowel (E) of RaraDN ${ }^{\text {LoxP/+; }}$ Sox $10 C r e^{+}$mice. Scale bars: $500 \mu \mathrm{m}$

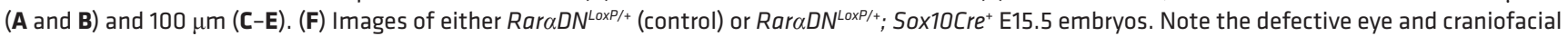

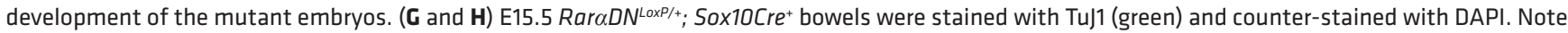

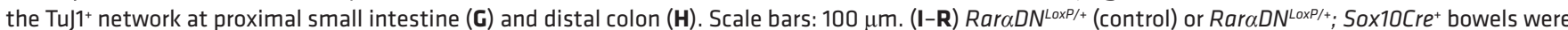
stained with HuC/D (red) and TuJ1 (green) antibodies. Representative images of esophagus (I and J), stomach (K and $\mathbf{L}$ ), proximal small intestine (M and $\mathbf{N})$, distal small intestine $(\mathbf{O}$ and $\mathbf{P})$, and end of distal colon (Q and $\mathbf{R})$. Scale bar: $50 \mu \mathrm{m}$. (S) Proximal small intestine of E15.5 RaraDN ${ }^{\mathrm{LoxP} /+} ;$ Sox10Cre ${ }^{+}$bowel. Regions outlined by boxes indicate which areas are enlarged in (T-V). Note extrinsic nerve fibers (T and $\mathbf{U})$. Scale bars: $500 \mu \mathrm{m}(\mathbf{S})$ and $50 \mu \mathrm{m}(\mathbf{T}-\mathbf{V})$. $n=3$,

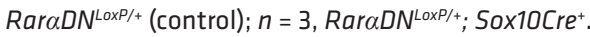

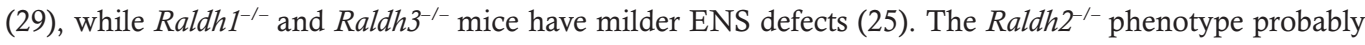
occurs because RA is needed in vagal paraxial mesoderm starting at E9 in mice (or E1.5 in avians) to induce Ret expression in ENCDC as these cells migrate from neural tube to bowel (24). In contrast, when RAR signaling was blocked by the chemical pan-RAR antagonist BMS493 at E11.5 in organ culture (26) or at E12.5 in dissociated cell culture (27), RET expression in ENCDC appeared unaffected by RAR inhibition. Nonetheless, RAR inhibition at these later stages impaired ENCDC proliferation, bowel colonization, neurite growth, neuronal differentiation, and ENS patterning $(23,26,27)$. Consistent with these observations, nutritional deficiency in the RA precursor vitamin A causes distal bowel aganglionosis in mice, mimicking human Hirschsprung disease (HSCR) (26), and gene variants in people with HSCR may alter RA signaling $(45,46)$. These studies suggest that RA signaling could impact ENS development in many ways, depending on the timing and severity of the RA signaling defect.

Prior strategies did not define the cell types influenced by RA signaling. To determine how cellautonomous RAR signaling affects the ENS lineage, we used a potent CRE-inducible dominant-negative RAR transgene (RARaT403) that blocks all 3 RAR receptors (i.e., Rar $\alpha N^{\text {toxP } /+}$ mice) (47). By breeding to several different CRE-expressing mouse lines, we induced RARaT403 (RAR $\alpha \mathrm{DN}$ ) expression selectively within ENCDC at specific times during development. Our RaraDN ${ }^{L o x P /+}$; Wnt $1 \mathrm{Cre}^{+}$studies show that cellautonomous RAR signaling within ENCDC is required to activate Ret expression early in development, consistent with data from zebrafish (21) and avian models (24). RAR signaling is also needed to activate Ret in the ureteric bud, suggesting that similar cell-autonomous mechanisms may control Ret expression in the kidney and developing ENS $(34,48)$. In contrast, when CRE expression is driven by Ret regulatory elements, RaraDN 

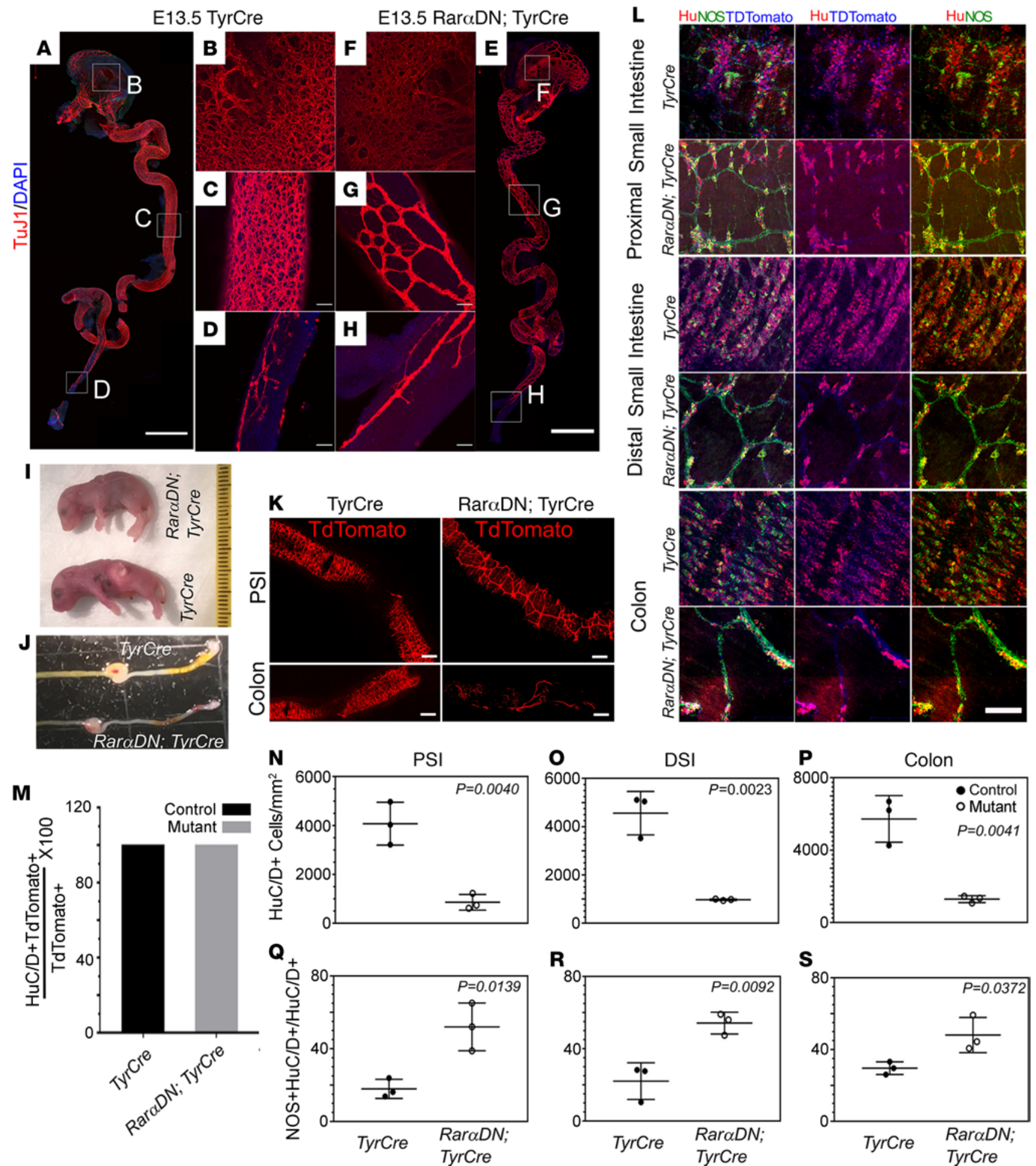

Figure 8. TyrCre-driven RaraDN reduces enteric neuron number and causes abnormal ENS patterning. (A-H) E13.5 RaraDN ${ }^{L o x P /+} ; T_{y r C r e}^{+}$or TyrCre $e^{+}$(control) bowels stained with Tuj1 antibody (red) and DAPI. Scale bars: $1 \mathrm{~mm}$ ( $\mathbf{A}$ and $\mathbf{E})$. Boxes in $\mathbf{A}$ and $\mathbf{E}$ indicate regions of stomach (B and $\mathbf{F})$, midgut (C and $\mathbf{G})$, and distal colon ( $\mathbf{D}$ and $\mathbf{H}$ ) that are magnified in adjacent images. Scale bars: $50 \mu \mathrm{m}(\mathbf{B}-\mathbf{H})$. (I) RaraDN ${ }^{L \times x P /+}$; TyrCre+ appear similar to control (TyrCre $)$ at birth but did not feed and lack a milk spot in stomach $(n=6)$. (J) RaraDN LoxP/+; TyrCre+ distal bowel appeared grossly similar to control (TyrCre+) at PO $(n=10)$. (K) TdTomato $^{+}$images of proximal small intestine (PSI) and colon of either RaraDN LoxP/+; TyrCre ${ }^{+}$or TyrCre (control) E18.5 pups. Scale bar: 1 mm. (L) Whole-mount IHC of E18.5 RaraDN ${ }^{\mathrm{LoxP} P+}$; TyrCre+ or TyrCre+ (control) bowels using HuC/D (red) and NOS1 (green) antibodies and via TdTomato (blue) fluorescence. Scale bar: 100

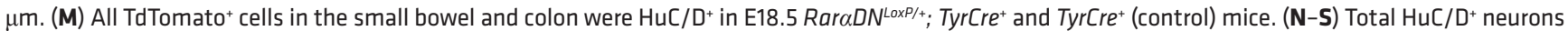
were reduced in PSI, distal small intestine (DSI) and colon of RaraDN ${ }^{L o x P /+} ;$ TyrCre ${ }^{+}$mice (N-P), but the percentage of HuC/ $\mathrm{D}^{+}$neurons expressing NOS1 was increased in mutants (Q-S). $n=3$, TyrCre+ (control); $n=3$, RaraDN ${ }^{\text {LoxP/++}}$; TyrCre+ . Two-tailed unpaired Student's $t$ tests were used for statistics. 
E13.5 RaraDN; RetCreERT2-EYFP Tandem Tamoxifen at E8.5
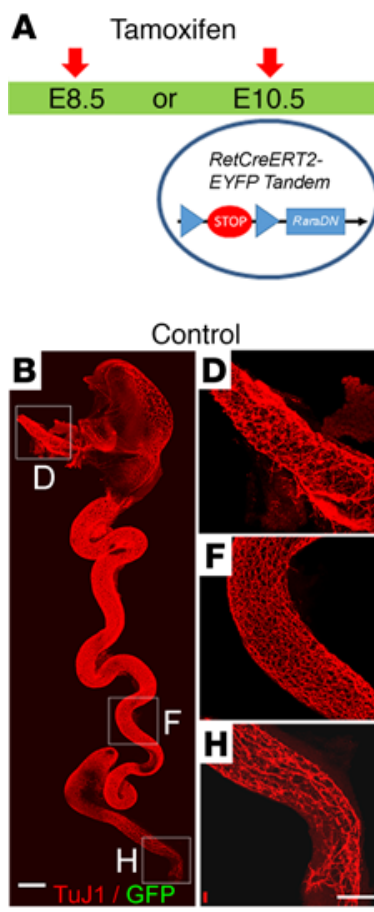

RaraDN; RetCreERT2-EYFP
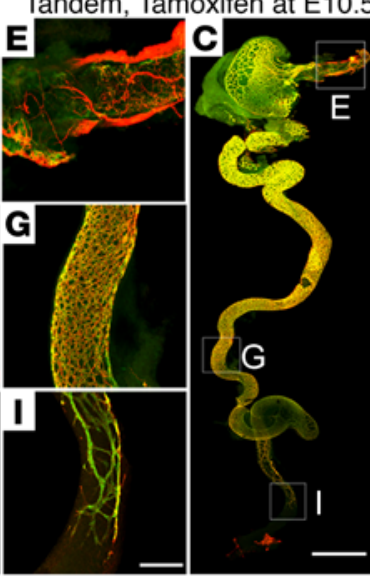

Harvest and analyze E13.5 RetCreERT2EYFP Tandem -1 RanON

Figure 9. Inactivation of RAR signaling in Ret lineage at E8.5 or E10.5. (A) Rar $\alpha D N^{L o x P /+} ; R E T C r e E R T 2-E Y F P^{T a n d e m}$ have CreERT2 knocked into the Ret locus and ChR2-Lox-Stop-Lox-EYFP in the nearby Rosa26 locus on the same chromosome. Tamoxifen activates CreERT2 to induce expression of RaraDN and EYFP. (B and C) E13.5 whole bowel from control (RaraDN LoxP/+ that lacks RETCreERT2-EYFPTandem) (B) RaraDN ${ }^{L 0 x P /+}$; RETCreERT2-EYFPTandem (C) after E10.5 tamoxifen treatment. ENS was visualized using TuJ1 (red) and GFP (green) antibodies. (D-I) Boxes indicate regions of esophagus (D and E), small bowel (F and $\mathbf{G}$ ), and distal colon ( $\mathbf{H}$ and $\mathbf{I}$ ) that are magnified in adjacent images. Scale bars: $1000 \mu \mathrm{m}$ (B and $\mathbf{C}$ ) and $500 \mu \mathrm{m}$ (D-I). (J and $\mathbf{K})$ E13.5 bowel after E8.5 tamoxifen treatment. Scale bar: $1000 \mu \mathrm{m}$. (L and M) Regions of magnified images from RaraDN LoxP/+; RETCreERT2-EYFPTandem small bowel (L) or distal colon (M). Scale bar: $100 \mu \mathrm{m}$. (N and $\mathbf{0})$ RET (red) and GFP (green) antibody stained E13.5 colon after E10.5 tamoxifen treatment. (P and Q) PHOX2B (green), GFP (red), and DAPI (blue) stained E13.5 colon after E10.5 tamoxifen treatment. Scale bars: $100 \mu \mathrm{m} . n=3$ in each group.

induction did not lead to loss of RET protein in ENCDC, suggesting that RA is needed to turn on Ret but not to maintain Ret expression, consistent with our prior BMS493 data $(26,27)$. We were surprised to discover that PHOX2B protein was also undetectable in ENCDC of RaraDN ${ }^{\mathrm{LoxP} /+}$; Wnt $1 \mathrm{Cre}^{+}$mice. Loss of PHOX2B could, by itself, explain the loss of RET in RaraDN $N^{\mathrm{LoxP} /+}$; Wnt1Cre+ ${ }^{+}$ENCDC (40), but we are unable to find evidence that $\mathrm{PHOX} 2 \mathrm{~B}$ regulates RET in the kidney, suggesting that Ret is regulated by RA via additional PHOX2B-independent mechanisms, as supported by prior studies $(30,45,49)$.

There were many other interesting observations. First, the thick chains of enteric neurons seen in the distal bowel of RaraDN $N^{L o x P /+}$; TyrCre ${ }^{+}$and RaraDN $N^{L o x P /+} ; R E T C r e E R T 2-E Y F P^{\text {Tandem }}$ mice closely resemble the colon ENS patterning defect we observed in BMS493-treated fetal gut organ cultures (26). This suggests that the normal dispersion of enteric neurons into small colon ganglia is RA dependent. Second, in fetal RaraDN LoxP/+ ; SOX10Cre+ mice, we saw some clustered small bowel enteric neurons near extrinsic nerve fibers but far from the more proximal ENS cells. These clusters closely resemble Schwann cell-derived ENS described by Uesaka et al., but the ENCDC we observed were restricted to small regions of fetal bowel (10). Alternatively, these cells might originate from the "mesenteric neural crest cells" recently described by Yu et al. that they hypothesize contribute to human skip segment HSCR (8). Third, vagus nerves at E11.5 in $\mathrm{RaraDN}^{\mathrm{LoxP} /+} ; \mathrm{Wnt} 1 \mathrm{Cre}^{+}$mice occupied a smaller area of the stomach compared with control animals. Since vagus nerve fibers were not TdTomato labeled in Wnt1Cre; R26R-TdTomato mice, this suggests reciprocal interactions between migrating Wnt1Cre lineage ENCDC and growing vagal fibers. Fourth, in addition to approximately $80 \%$ reduction in total neuron number in the ENS of RaraDN ${ }^{\mathrm{LoxP} /+}$; $\mathrm{TyrCre}^{+}$mice, there was a striking increase in the percentage of enteric neurons that express NOS1 (nitric oxide synthase). We confirmed that these $\mathrm{NOS}^{+}$neurons had expressed CRE using an R26R-TdTomato reporter. This suggests that $\mathrm{NOS}^{+}{ }^{+}$neuron differentiation is less dependent on RAR signaling than other neuron subtypes or that RAR turns off Nos 1 expression in some enteric neuron subtypes. Finally, we found an almost complete loss of submucosal neurons in $\operatorname{RaraDN}^{\mathrm{LoxP} /+} ; R_{E T C r e E R T 2-E Y F P^{\text {Tandem }}}$ mice treated 

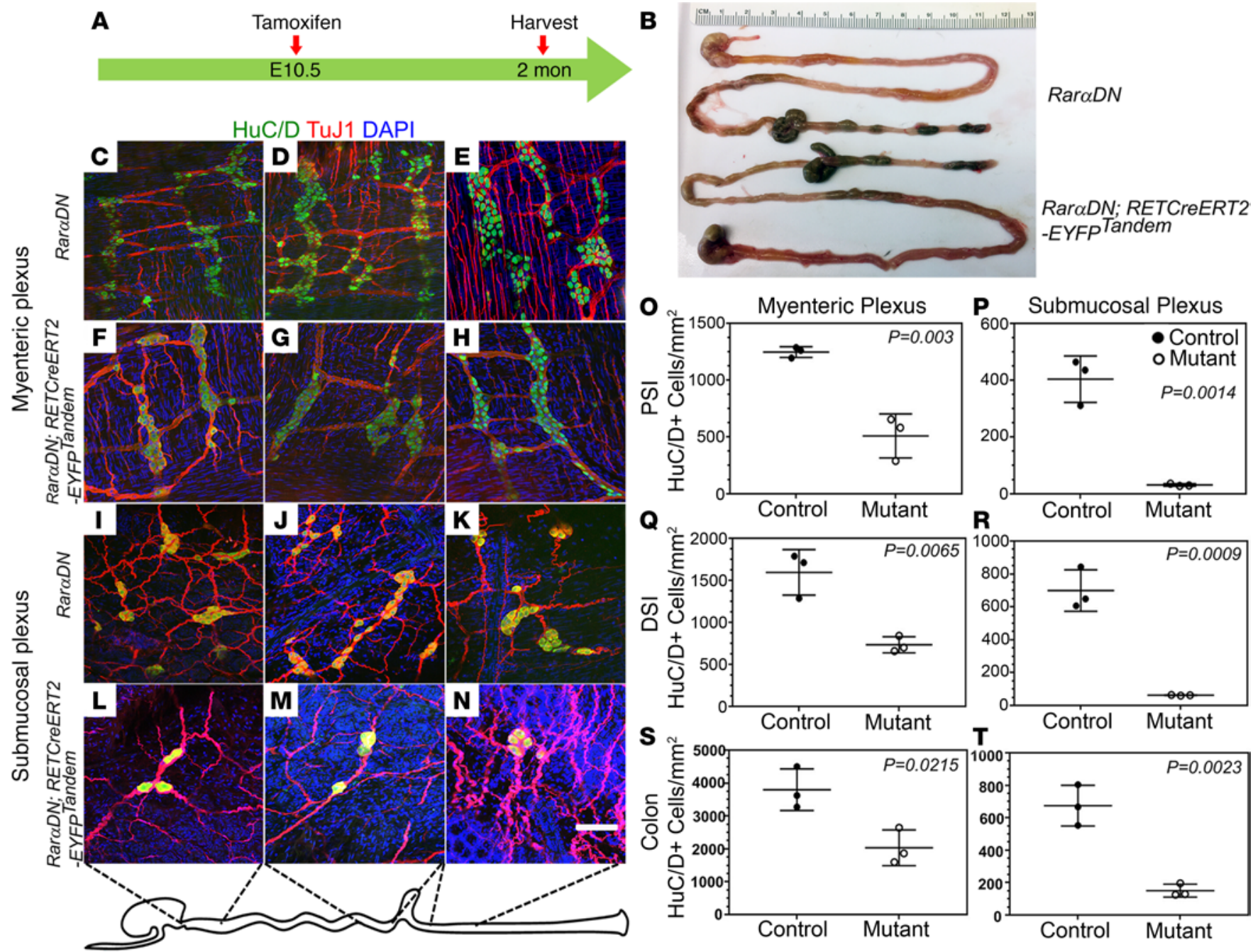

Figure 10. Postnatal ENS phenotype when RAR signaling is inactivated in Ret lineage starting from E10.5. (A) Tamoxifen was given to pregnant mice at E10.5 to activate the RaraDN transgene, and bowel was harvested at about 2 months of age. (B) RaraDN LoxP/+ (control) or RaraDN ${ }^{L \alpha \times P /+} ; R E T C r e E R T 2-E Y-$

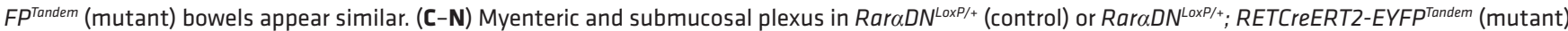
animals were visualized in whole mounts using HuC/D (green) and TuJ1 (red) antibodies plus DAPI (blue). Scale bar: $100 \mu \mathrm{m}$. (O-T) Quantification of HuC/ $D^{+}$neurons shows about 50\% fewer myenteric and about 90\% fewer submucosal neurons in RaraDN-RETCreERt2-EYFPTandem (mutant) animals compared with RaraDN ${ }^{L x P /+}$ (control) ( $n=3$ in each group). Two-tailed unpaired Student's $t$ tests were used for statistics.

with tamoxifen at E10.5, suggesting a critical role for RA signaling in radial migration of ENCDC to form submucosal plexus. The normal postnatal growth of these tamoxifen-treated RaraDN ${ }^{\text {LoxP/+ }} ;$ RETCreERT2-EYFP Tandem mice and normal appearance of the adult bowel suggests that a loss of approximately $90 \%$ of submucosal neurons is well tolerated, at least in mice. This is interesting, in part, because we know little about mechanisms controlling radial migration of ENS precursors to the submucosal plexus, with prior studies implicating only GDNF and netrin/DCC signaling in this process $(50,51)$. These observations highlight the remarkable range of ENS abnormalities that may occur when RAR signaling is inadequate and are consistent with the observation that low serum vitamin A is associated with increased constipation in children with autism (52).

One concern is that we did not evaluate ENS biology in every possible control group, so we cannot exclude some effects of tamoxifen, Cre alleles, fluorescent reporters, or the $R a r \alpha D N^{L o x P /+}$ allele in isolation. For

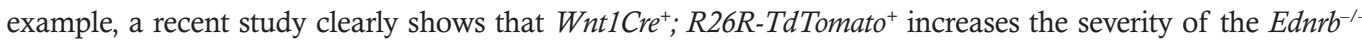
ENS phenotype (8). We also were underpowered to examine the effect of sex on ENS phenotypes, an issue that could be important for some of the milder phenotypes we examined.

One advantage of our strategy is that CRE-dependent expression of RAR $\alpha \mathrm{DN}$ and a fluorescent reporter in the same cells facilitated flow sorting and RNA-seq to identify RAR targets. Our analyses identified thousands of differentially expressed genes in RAR $\alpha \mathrm{DN}$-expressing versus control ENCDC at E11.5 and E13.5 
A

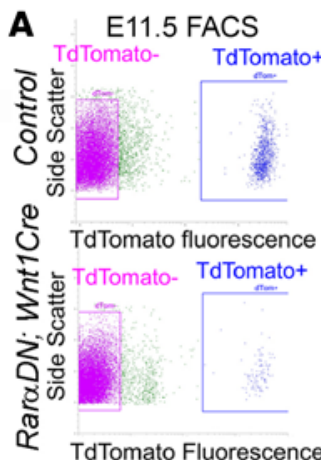

B

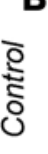

হ.

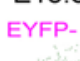

E13.5

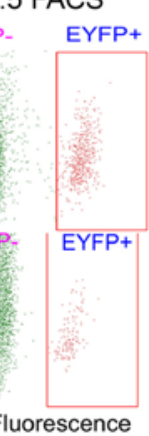

EYFP Fluorescence

E11.5

E Up-regulated in mutant Down-regulated in mutant

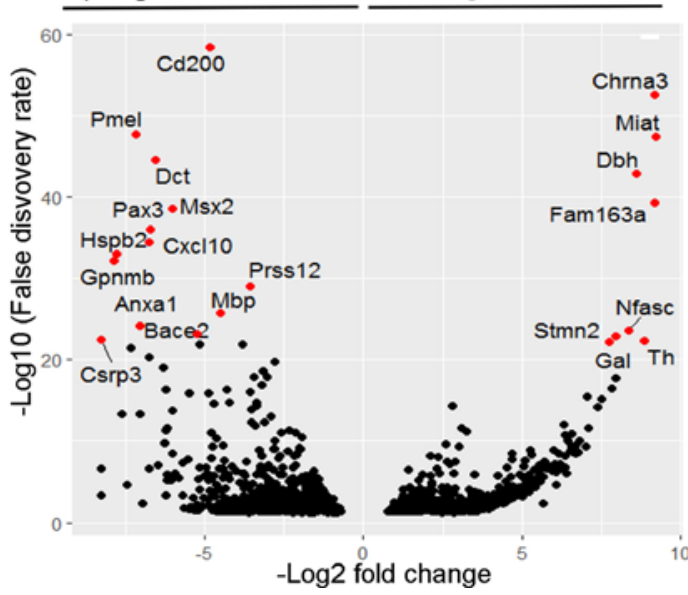

C $\quad \mathrm{E} 11.5$

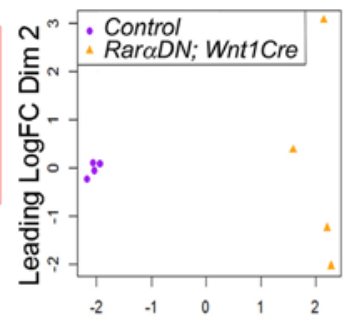

Leading LogFC Dim 1
D $\quad \mathrm{E} 13.5$

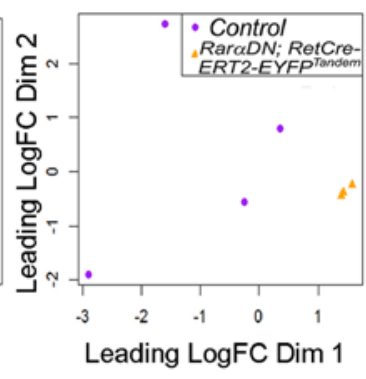

E13.5

F Up-regulated in mutant Down-regulated in mutant

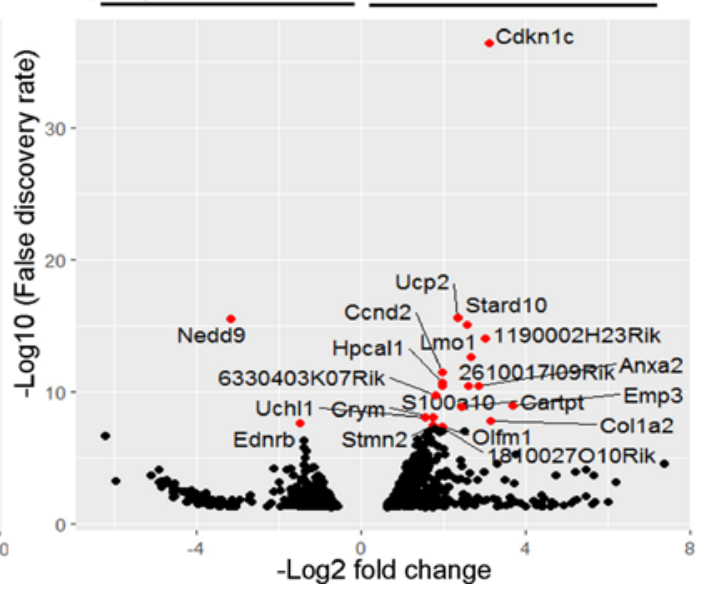

Figure 11. RNA-seq shows that many genes are dysregulated after RaraDN expression in the ENS. (A) ENCDC were isolated from E11.5 stomach of RaraDN LoxP/+; Wnt1Cre+; R26R-TdTomato and Wnt1Cre+; R26R-TdTomato (control) mice based on TdTomato fluorescence ( $n=4$ each). (B) ENCDC were isolated from E13.5 colon of RaraDNLoxP/+; RETCreERT2-EYFP Tandem or RETCreERT2-EYFPTandem (control) mice based on EYFP fluorescence after tamoxifen treatment at E10.5 ( $n=4$ each, but 1 RaraDN ${ }^{\text {LxP/++}}$; RETCreERT2-EYFPTandem failed quality control, leaving $n=3$ for this genotype). (C and D) Multidimensional scaling analysis (MDS) of RNA-seq data. (E and F) Volcano plots show differentially expressed genes.

with 115 genes regulated in the same way by $\mathrm{RAR} \alpha \mathrm{DN}$ at each age. Interpreting these data is complicated, since $\mathrm{RAR} \alpha \mathrm{DN}$ induces changes in cell type ratios, in addition to changes in gene expression in individual cells. Furthermore, it is not clear if the induced changes occur because of direct effects on RAREs or if they reflect more global effects on the differentiation state of the sequenced cells. For example, loss of PHOX2B in E11.5 ENCDC should change the expression of many genes, independent of effects on RAR activity.

As one strategy to further define the role of RAR signaling, we narrowed our lists to include only differentially expressed genes (FDR < 0.2) where expression changed in the same direction at E11.5 and E13.5 for $\mathrm{RAR} \alpha \mathrm{DN}^{+}$cells (Supplemental Tables 4 and 5). At E11.5, there were 55 genes expressed at higher levels in WT ENCDC than in RAR $\alpha \mathrm{DN}^{+}$ENCDC. Thirty-five of these genes had easily identified functions in neurons. Thirteen genes at E11.5 were more abundant in $\mathrm{RAR} \alpha \mathrm{DN}^{+} \mathrm{ENCDC}$ than in WT ENCDC. This list included Rest, an epigenetic master negative regulator of neurogenesis $(53,54)$, and Bmp4, a gene with complex roles in ENS patterning (55-62). At E13.5, our list included 44 genes more abundant in WT than in $\mathrm{RAR} \mathrm{DN} \mathrm{N}^{+}$cells. Twenty-nine of these genes had easily identified roles in neurons. Genes more abundant in $\mathrm{RAR} \alpha \mathrm{DN}^{+}$than in WT cells at E13.5 included Rest, Sox10, and Ets1, consistent with a role for RAR in ENS neurogenesis. The SOX10 transcription factor is essential for ENS development (63), at least in part because SOX10 activates RET expression $(64,65)$. However, as multipotent ENCDC differentiate into enteric neurons, SOX10 expression is lost, while enteric glia continue to express SOX10 through adulthood (3). ETS1 enhances Sox10 expression and is essential to make radial glia in Xenopus (66). Consistent with these mRNA findings, SOX10 ${ }^{+}$cells were abundant in myenteric and submucosal plexus of adult RaraDN-expressing mice, even though $\operatorname{Rar} \alpha D N^{L o x P /+}$; RETCreERT2-EYFP Tandem had an approximately $90 \%$ reduction in submucosal neurons and the enteric glia are 
A
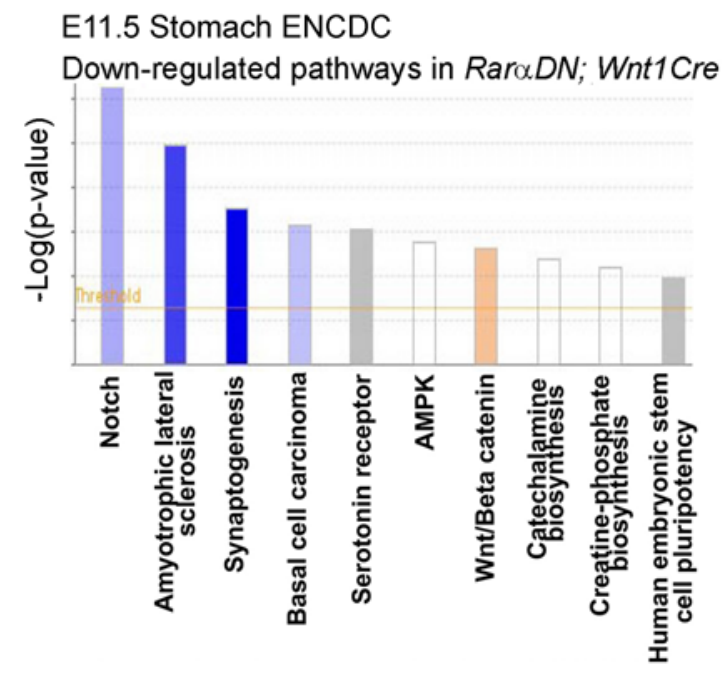

Up-regulated pathways in RaroDN; Wnt1Cre

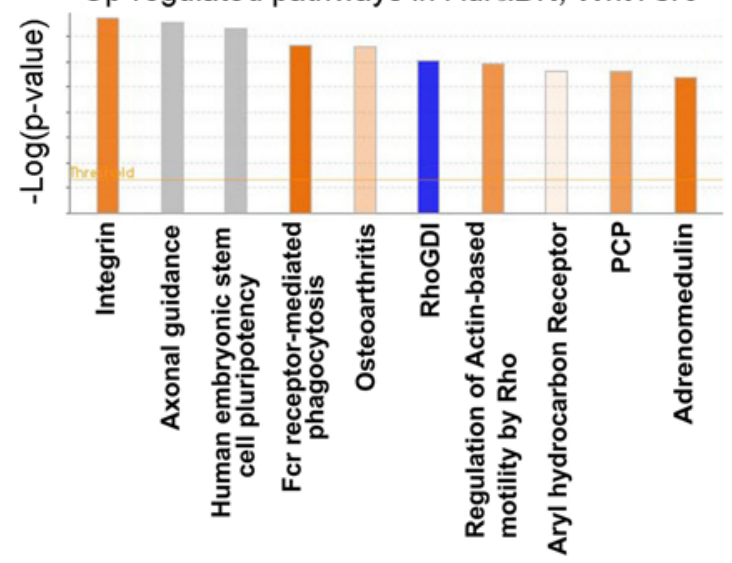

B

\section{E13.5 Stomach ENCDC \\ Down-regulated pathways in Rar $\alpha D N$; RETCreERT2- EYFPTandem}

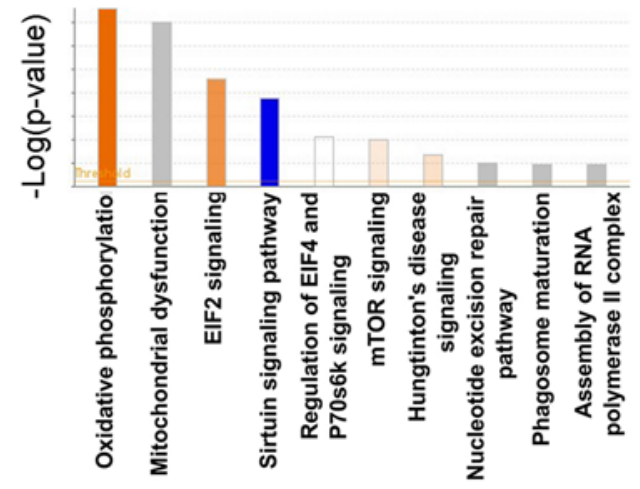

Up-regulated pathways in Rar $\alpha D N$; RETCreERT2EYFPTandem

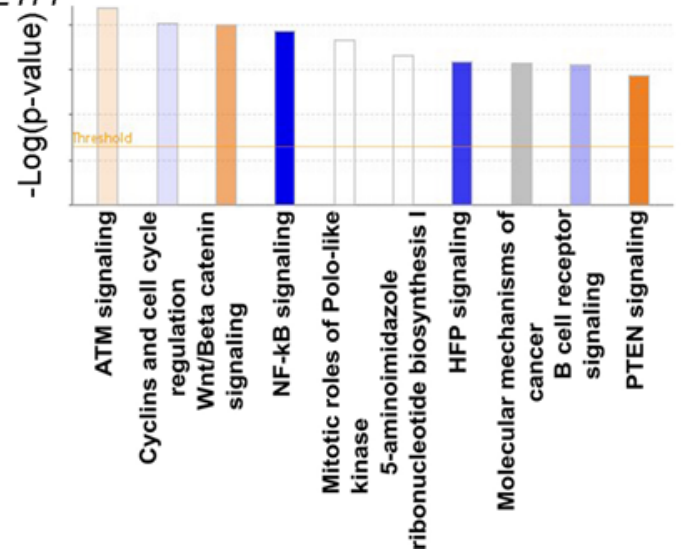

Figure 12. Pathway analysis for genes dysregulated by RAR $\alpha$ DN. (A and B) Ingenuity Pathway Analysis (IPA) of differentially expressed genes (FDR < $0.05)$ at E11.5 (A) and E13.5 (B).

derived from RET ${ }^{+}$ENCDC (67). In addition, many Wnt ligands (Wnt1, Wnt2, Wnt4, and Wnt10a) were highly differentially expressed in E11.5 ENCDC of RaraDN ${ }^{L-o x P /+}$; Wnt $1 \mathrm{Cre}^{+}$versus control. Since Wnt signaling plays important roles in many aspects of neural development (68-70), RAR might alter Wnt signaling to regulate ENCDC development. Collectively, these data suggest that cell-autonomous RAR signaling directs neurogenesis in the ENS from multipotent ENCDC and that cell-autonomous RAR signaling has distinct effects at many stages of ENS development. Our observations further suggest that maternal retinoid status during pregnancy and postnatal vitamin A deficiency or excess could have long-term effects on ENS structure and function. This may be important because vitamin A deficiency is a common problem in many regions of the world (71).

\section{Methods}

Mouse strains. RaraDN Loxp-STOP-LoxP/+ (previously described in ref. 34) were rederived from sperm into C57BL/6J background by the CHOP transgenic core. H2az2(Tg[Wnt1cre]11Rth) (Research Resource Identifiers [RRID]: 2386570_JAX:003829; referred to as Wnt1Cre), TyrCre (RRID: MGI:358524_JAX:029788), SOX10Cre (MGI:3586900_JAX:025807), Rosa26 ${ }^{\text {EYFP }}$ (Gt[ROSA]26Sortm1[EYFP]Cos, MGI: 2449038, RRID:IMSR_EM: 09668), and Gt(ROSA)26Sortm9(CAGtdTomato)Hze (RRID:IMSR_JAX:007909; referred to as R26R-TdTomato) were from The Jackson Laboratory and maintained in C57BL/6J background. RETCreERT2-EYFPTandem mice, a gift from Wenqin Luo (University of Pennsylvania), were generated by breeding RETCreERT2 (RRID: MGI:4437245) to mice with ChR2-Lox-Stop-Lox-EYFP in the Rosa26 locus (72). Mouse Ret and Rosa26 loci are close together (about 5 million bp), but RETCreERT2-EYFP Tandem recombined so that they are on the same chromosome. $\operatorname{Ret}^{T G M / T G M}\left(\operatorname{Ret}^{-1-}\right.$ allele $\operatorname{Ret}^{\mathrm{tm} I J m i}$ ) on C57BL/6J were previously described (73). A complete list 
of mouse strains appears in Supplemental Table 6, Genotyping was performed using previously published and potentially novel primers (Supplemental Table 7).

Mouse husbandry. Mice had a 12-hour light-dark cycle with free access to food and water. Additional details based on ARRIVE guidelines (74) are in Supplemental Table 8. For timed pregnant mating, Rar $\alpha D$ female mice were bred with Cre-expressing male mice in the late afternoon. Successful mating was confirmed by vaginal plug the next morning, which was designated E0.5.

EdU incorporation assay. EdU analyses were performed using a Click-iT EdU imaging kit (Thermo Fisher Scientific, C10337). Timed pregnant animals (E12.5) were injected (i.p.) with EdU (12.5 $\mu \mathrm{g} / \mathrm{gm}$ body weight). Tissues were dissected 4 hours after EdU and fixed (4\% paraformaldehyde [PFA], 30 minutes at room temperature) before staining, using manufacturer's protocol.

Whole mouse embryo imaging using 3DISCO clearing. 3DISCO imaging was performed as described (38) with modification. Briefly, E10.5 embryos from timed-pregnant females were washed in PBS ( $\mathrm{pH} 7.4$ ), fixed (4\% PFA, 1 hour, room temperature), and then incubated in SOX10 (1:200, Novus Biologicals, AF2864) and TuJ1 (1:2000 dilution, Covance, PRB-435P, RRID:AB_2313773) antibodies in $1 \times$ PBS with $0.5 \%$ triton X-100 (PBST) and 5\% normal donkey serum (NDS) $\left(4^{\circ} \mathrm{C}\right.$, constant rocking, 72 hours). After washing 4 times (PBS, room temperature, 15 minutes per wash), embryos were incubated in secondary antibodies and Hoechst 33342 for nuclear staining ( $2 \mu \mathrm{g} / \mathrm{mL}$, Invitrogen, catalog $\mathrm{H} 3570)$ in PBST with $5 \%$ NDS ( $4^{\circ} \mathrm{C}$, overnight) and then washed 4 times (PBS, room temperature, 15 minutes per wash) and subjected to 3DISCO clearing using a series of tetrahydrofuran (MilliporeSigma, 401757) incubations (room temperature, $50 \%, 70 \%$, and then $80 \%$ in double-distilled water, 60 minutes each, with rocking), followed by incubation in 100\% tetrahydrofuran (3 $\times 20$ minutes, room temperature, rocking). Embryos were then incubated overnight in dibenzyl either (MilliporeSigma, 108014-1KG) at room temperature and mounted in dibenzyl ether before confocal imaging.

$I H C$. For whole-mount staining, fetal bowel was fixed as a tube. Adult bowels were opening along mesentery and pinned flat to Sylgard before fixation. Bowels were fixed with 4\% PFA (40 minutes, room temperature, rocking), washed ( $4 \times 10$ minutes, PBS, room temperature), blocked ( $5 \%$ NDS in PBST, room temperature, 1 hour), and then incubated overnight $\left(4^{\circ} \mathrm{C}\right)$ with primary antibodies in $5 \%$ NDS in PBST. Secondary antibody staining, unless otherwise specified, was done by incubating samples in a 1:400 dilution of the appropriate antibody $\left(1\right.$ hour, $\left.25^{\circ} \mathrm{C}\right)$. All the primary and secondary antibodies are listed in Supplemental Table 9.

$R N A$-seq analyses. E11.5 stomach ENCDC were isolated from RaraDN ${ }^{L o x P /+} ;$ Wnt1Cre ${ }^{+}$R26R-TdTomato and $W n t 1 \mathrm{Cre}^{+}$; R26R-TdTomato (control) mice. E13.5 colon ENCDC were isolated from RaraDN ${ }^{L o x P /+}$; RETCreERT2-EYFP Tandem or RETCreERT2-EYFP Tandem (control) mice after treatment with tamoxifen (oral gavage, 233 $\mathrm{mg} / \mathrm{kg}$ body weight dissolved in corn oil) at E10.5. Individual E11.5 stomach and E13.5 colon were incubated with collagenase (MilliporeSigma, C0130, $0.2 \mathrm{mg} / \mathrm{mL}$ ) and dispase (MilliporeSigma, 494207800, $0.2 \mathrm{mg} / \mathrm{mL}$ ) in Ham's F12 media (Thermo Fisher Scientific, 11765054) with $1 \% \mathrm{BSA}\left(37^{\circ} \mathrm{C}, 5 \% \mathrm{CO}_{2}\right.$ incubator, 20 minutes) and then triturated by pipetting up and down 15 times using a P1000 pipette tip before passing through a $30 \mu \mathrm{m}$ strainer (Corning, 352340). Cells with TdTomato or EYFP fluorescence were purified by flow sorting (MoFlo Astrios high speed cell sorter, $100 \mu \mathrm{m}$ nozzle), pelleted by centrifugation $\left(500 \mathrm{~g}, 4^{\circ} \mathrm{C}, 5\right.$ minutes $)$, washed once with $\mathrm{PBS}$, and frozen immediately at $-80^{\circ} \mathrm{C}$ until analysis. RNA isolation, library preparation, and RNA-seq analysis were performed by University of Pennsylvania Next-Generation Sequencing Core. RNA extracted using the GenElute Single Cell RNA Purification Kit (MilliporeSigma, RNB300) was analyzed on a 2100 Agilent Bioanalyzer using an RNA 6000 Pico Kit (Agilent, 5067-1513). The libraries were made using the combination of the Clontech (now Takara), SMART (cDNA synthesis and amplification), and Illumina Nextera XT (cDNA to library) kits. Libraries were then single-end sequenced on an Illumina HiSeq 4000, using Sequencer Software Real-Time Analysis (RTA) 2.7.7 and Illumina HiSeq Control Software (HCS) HD 3.4.0.38. Reads were aligned to remove repeat sequences and ribosomal RNA reads and then processed using RNA-seq unified mapper (RUM) package (75). RUM files were visualized in the TessLA browser for down-stream analyses. MDS analysis, volcano plot, and heatmaps were made on R 4.0.2 platform using limma, edgeR, Glimma, gplots, org.Mm.eg.db, and RColorBrewer. We used ggrepel, ggplot2, and reshape to generate Volcano plots, and we used tidyr, gplots, and ggplot2 to generate heatmaps. Gene expression pathway analyses were performed using Ingenuity Pathway Analysis (IPA) package (Qiagen) and Metascape Gene Annotation \& Analysis Resource (76). A Venn diagram was generated using the Draw Venn Diagram software of Bioinformatics \& Evolutionary Genomics, VIB/UGent, Belgium. The RNA-seq raw data have been deposited in the GEO NIH data repository under the accession no. GSE165344.

Real time PCR. E11.5 stomach ENCDC of RaraDNLoxP/+; Wnt1Cre ${ }^{+}$R26R-TdTomato and Wnt1Cre ${ }^{+}$R26R-TdTomato (control) mice were isolated via the same protocol as for RNA-seq analysis. RNA was purified using 
PicoPure RNA isolation kit (Arcturus, KIT0202). cDNA was made by using First-strand cDNA synthesis using superscript II RT kit (Invitrogen, 18064-022) with Oligo(dT)12-18 primer (Invitrogen, 18418012). Quantitative PCR (qPCR) was performed using SYBR Green qPCR mixture (MilliporeSigma, S4438). All primers have been previously described and listed in Supplemental Table 10. Ct values were normalized to Gapdh mRNA.

Confocal microscopy. High-resolution 3-dimensional images were acquired with a Zeiss LSM 710 confocal microscope using Zeiss ZEN 2.3 SP1 FP3 (black) (Version 14.0.18.201; Zeiss) software and a 20×/0.8 air or $63 \times / 1.4$ oil DIC M27 Plan-Apochromat objective, except for whole bowel E12.5 and E13.5 imaging, which employed a $5 \times$ objective and the ZEN Tile Scan function. Image stitching employed ZEN software. Z-stacks were created with ImageJ/FIJI. Increments between each slice were $1 \mu \mathrm{m}$, except for scanning the whole E10.5 embryo, where increments between slices were $3 \mu \mathrm{m}$. Videos of $Z$-stacks were created with the animation function of Imaris 9.02 .

Image analysis. Early embryonic, perinatal, and adult mouse bowel cell counting used $>5$ randomly selected $\times 20$ fields per animal in each region and FIJI CellCounter module (NIH). Investigators were blinded to genotype when comparing RaraDN mutant and control mice.

Statistics. All experiments include at least 3 replicates. Statistical analyses employed GraphPad Prism 7 software. Data normality analyses employed the Shapiro-Wilk test. Comparisons between groups used 2-tailed unpaired Student's $t$ tests. $P<0.05$ is considered statistically significant. Data show mean \pm SEM.

Study approval. Mouse studies were performed with approval from the Institutional Use and Care Committee at The Children's Hospital of Philadelphia (CHOP) Research Institute (IAC 19-001041) and the Animal Studies Committee of Washington University School of Medicine in St. Louis (no. 20120130).

\section{Author contributions}

TG and ROH led this study and wrote the manuscript. TG, ECWJ, RS, JBA, and ROH designed research studies, conducted experiments, acquired data, analyzed data, and edited the manuscript.

\section{Acknowledgments}

We thank Cathy L. Mendelsohn (Columbia University) for generously sharing her Rar $\alpha D N^{L o x P /+}$ mice and her insight into retinoid biology. We also thank Wenqin Luo for generously sharing RETCreERT2-EYFPTandem mice. This work was supported by the Irma and Norman Braman Endowment (to ROH), the Suzi and Scott Lustgarten Center Endowment (to ROH), the Children's Hospital of Philadelphia Research Institute (to ROH), Children's Discovery Institute of Washington University and Saint Louis Children's Hospital (MD-II2013-269) (to ROH), R01 DK087715 (to ROH), March of Dimes 6-FY15-235 (to ROH), and the Burroughs Wellcome Fund Clinical Scientist Award in Translational Research (1008525) (to ROH). We greatly appreciate insightful discussions with Paul Trainor. We thank the CHOP Flow Cytometry Core and Next-Generation Sequencing Core at The University of Pennsylvania for assisting us with the RNA-seq experiment. CHOP Transgenic Core helped us rederive the RaraDN mice.

Address correspondence to: Robert O. Heuckeroth, The Children's Hospital of Philadelphia, Research Institute, 3615 Civic Center Blvd, Abramson Research Center - Suite \# 1116I, Philadelphia, Pennsylvania 19104-4318, USA. Phone: 215.590.1209; Email: HeuckerothR@chop.edu. RS’s present address is: American Association for Cancer Research. ECWJ's present address is: Department of Neurology, Nemours, Alfred I. Dupont Hospital for Children.

1. Schneider S, et al. Unexpected roles for the second brain: enteric nervous system as master regulator of bowel function. Annu Rev Physiol. 2019;81:235-259.

2. Furness JB. The enteric nervous system and neurogastroenterology. Nat Rev Gastroenterol Hepatol. 2012;9(5):286-294.

3. Lake JI, Heuckeroth RO. Enteric nervous system development: migration, differentiation, and disease. Am J Physiol Gastrointest Liver Physiol. 2013;305(1):G1-24.

4. Uesaka T, et al. Development of the intrinsic and extrinsic innervation of the gut. Dev Biol. 2016;417(2):158-167.

5. Sasselli V, et al. The enteric nervous system. Dev Biol. 2012;366(1):64-73.

6. Avetisyan M, et al. Building a second brain in the bowel. J Clin Invest. 2015;125(3):899-907.

7. Burns AJ, Le Douarin NM. The sacral neural crest contributes neurons and glia to the post-umbilical gut: spatiotemporal analysis of the development of the enteric nervous system. Development. 1998;125(21):4335-4347.

8. Yu Q, et al. Mesenteric neural crest cells are the embryological basis of skip segment Hirschsprung's disease [published online December 16, 2020]. Cell Mol Gastroenterol Hepatol. https://doi.org/10.1016/j.jcmgh.2020.12.010.

9. Espinosa-Medina I, et al. Dual origin of enteric neurons in vagal Schwann cell precursors and the sympathetic neural crest. Proc Natl 
Acad Sci U S A. 2017;114(45):11980-11985.

10. Uesaka T, et al. Neuronal differentiation in schwann cell lineage underlies postnatal neurogenesis in the enteric nervous system. J Neurosci. 2015;35(27):9879-9888

11. Brokhman I, et al. Dual embryonic origin of the mammalian enteric nervous system. Dev Biol. 2019;445(2):256-270.

12. Drokhlyansky E, et al. The human and mouse enteric nervous system at single-cell resolution. Cell. 2020;182(6):1606-1622.

13. May-Zhang AA, et al. Combinatorial transcriptional profiling of mouse and human enteric neurons identifies shared and disparate subtypes in situ. Gastroenterology. 2021;160(3):755-770.

14. Zeisel A, et al. Molecular architecture of the mouse nervous system. Cell. 2018;174(4):999-1014.

15. Morarach K, et al. Diversification of molecularly defined myenteric neuron classes revealed by single-cell RNA sequencing. Nat Neurosci. 2021;24(1):34-46.

16. Memic F, et al. Transcription and signaling regulators in developing neuronal subtypes of mouse and human enteric nervous system. Gastroenterology. 2018;154(3):624-636.

17. Wright CM, et al. scRNA-Seq reveals new enteric nervous system roles for GDNF, NRTN, and TBX3 [published online January 11, 2021]. Cell Mol Gastroenterol Hepatol. https://doi.org/10.1016/j.jcmgh.2020.12.014.

18. Foong JP. Postnatal development of the mouse enteric nervous system. Adv Exp Med Biol. 2016;891:135-143.

19. Yarandi SS, et al. Intestinal bacteria maintain adult enteric nervous system and nitrergic neurons via toll-like receptor 2-induced neurogenesis in mice. Gastroenterology. 2020;159(1):200-213.

20. Kulkarni S, et al. Adult enteric nervous system in health is maintained by a dynamic balance between neuronal apoptosis and neurogenesis. Proc Natl Acad Sci U S A. 2017;114(18):E3709-E3718.

21. Uribe RA, et al. Retinoic acid temporally orchestrates colonization of the gut by vagal neural crest cells. Dev Biol. 2018;433(1):17-32.

22. Suzuki R, et al. Abnormal neural crest innervation in Sox10-Venus mice with all-trans retinoic acid-induced anorectal malformations. Pediatr Surg Int. 2014;30(2):189-195.

23. Gisser JM, et al. A novel bidirectional interaction between endothelin-3 and retinoic acid in rat enteric nervous system precursors PLoS One. 2013;8(9):e74311.

24. Simkin JE, et al. Retinoic acid upregulates ret and induces chain migration and population expansion in vagal neural crest cells to colonise the embryonic gut. PLoS One. 2013;8(5):e64077.

25. Wright-Jin EC, et al. Retinaldehyde dehydrogenase enzymes regulate colon enteric nervous system structure and function. Dev Biol. 2013;381(1):28-37.

26. Fu M, et al. Vitamin A facilitates enteric nervous system precursor migration by reducing Pten accumulation. Development. 2010;137(4):631-640.

27. Sato Y, Heuckeroth RO. Retinoic acid regulates murine enteric nervous system precursor proliferation, enhances neuronal precursor differentiation, and reduces neurite growth in vitro. Dev Biol. 2008;320(1):185-198.

28. Pitera JE, et al. Embryonic gut anomalies in a mouse model of retinoic Acid-induced caudal regression syndrome: delayed gut looping, rudimentary cecum, and anorectal anomalies. Am J Pathol. 2001;159(6):2321-2329.

29. Niederreither K, et al. The regional pattern of retinoic acid synthesis by RALDH2 is essential for the development of posterior pharyngeal arches and the enteric nervous system. Development. 2003;130(11):2525-2534.

30. Cheng B, et al. Vitamin A deficiency exacerbates autism-like behaviors and abnormalities of the enteric nervous system in a valproic acid-induced rat model of autism. Neurotoxicology. 2020;79:184-190.

31. Frith TJR, et al. Retinoic acid accelerates the specification of enteric neural progenitors from in-vitro-derived neural crest. Stem Cell Reports. 2020;15(3):557-565.

32. Tan M, et al. Maternal vitamin A deficiency impairs cholinergic and nitrergic neurons, leading to gastrointestinal dysfunction in rat offspring via RARb. Life Sci. 2021;264:118688.

33. di Masi A, et al. Retinoic acid receptors: From molecular mechanisms to cancer therapy. Mol Aspects Med. 2015;41:1-115.

34. Rosselot C, et al. Non-cell-autonomous retinoid signaling is crucial for renal development. Development. 2010;137(2):283-292.

35. Danielian PS, et al. Modification of gene activity in mouse embryos in utero by a tamoxifen-inducible form of Cre recombinase. Curr Biol. 1998;8(24):1323-1326.

36. Gage PJ, et al. Fate maps of neural crest and mesoderm in the mammalian eye. Invest Ophthalmol Vis Sci. 2005;46(11):4200-4208.

37. Lake JI, et al. Hirschsprung-like disease is exacerbated by reduced de novo GMP synthesis. J Clin Invest. 2013;123(11):4875-4887.

38. Erturk A, et al. Three-dimensional imaging of solvent-cleared organs using 3DISCO. Nat Protoc. 2012;7(11):1983-1995.

39. Schuchardt A, et al. Defects in the kidney and enteric nervous system of mice lacking the tyrosine kinase receptor Ret. Nature. 1994;367(6461):380-383.

40. Pattyn A, et al. The homeobox gene Phox $2 \mathrm{~b}$ is essential for the development of autonomic neural crest derivatives. Nature. 1999;399(6734):366-370.

41. Debbache J, et al. Cre-driver lines used for genetic fate mapping of neural crest cells in the mouse: An overview. Genesis. 2018;56(6-7):e23105.

42. Fu M, et al. Retinoblastoma protein prevents enteric nervous system defects and intestinal pseudo-obstruction. J Clin Invest. 2013;123(12):5152-5164.

43. Fonseca-Pereira D, et al. The neurotrophic factor receptor RET drives haematopoietic stem cell survival and function. Nature. 2014;514(7520):98-101.

44. Canete A, et al. Role of vitamin A/retinoic acid in regulation of embryonic and adult hematopoiesis. Nutrients. 2017;9(2):E159.

45. Chatterjee $\mathrm{S}$, et al. Enhancer variants synergistically drive dysfunction of a gene regulatory network in hirschsprung disease. Cell. 2016;167(2):355-368.

46. Tang CS, et al. Uncovering the genetic lesions underlying the most severe form of Hirschsprung disease by whole-genome sequencing. Eur J Hum Genet. 2018;26(6):818-826.

47. Damm K, et al. Functional inhibition of retinoic acid response by dominant negative retinoic acid receptor mutants. Proc Natl Acad Sci U S A. 1993;90(7):2989-2993.

48. Batourina E, et al. Vitamin A controls epithelial/mesenchymal interactions through Ret expression. Nat Genet. 2001;27(1):74-78.

49. Angrisano T, et al. Chromatin and DNA methylation dynamics during retinoic acid-induced RET gene transcriptional activation 
in neuroblastoma cells. Nucleic Acids Res. 2011;39(6):1993-2006.

50. Jiang Y, et al. Netrins and DCC in the guidance of migrating neural crest-derived cells in the developing bowel and pancreas. Dev Biol. 2003;258(2):364-384

51. Uesaka T, et al. GDNF signaling levels control migration and neuronal differentiation of enteric ganglion precursors. $J$ Neurosci. 2013;33(41):16372-16382.

52. Cheng B, et al. Vitamin A deficiency increases the risk of gastrointestinal comorbidity exacerbates core symptoms in children with autism spectrum disorder. Pediatr Res. 2021;89(1):211-216.

53. Hwang JY, Zukin RS. REST, a master transcriptional regulator in neurodegenerative disease. Curr Opin Neurobiol. 2018;48:193-200 54. Zhao Y, et al. Brain REST/NRSF is not only a silent repressor but also an active protector. Mol Neurobiol. 2017;54(1):541-550.

55. Chalazonitis A, Kessler JA. Pleiotropic effects of the bone morphogenetic proteins on development of the enteric nervous system Dev Neurobiol. 2012;72(6):843-856.

56. Chalazonitis A, et al. Homeodomain interacting protein kinase 2 regulates postnatal development of enteric dopaminergic neurons and glia via BMP signaling. J Neurosci. 2011;31(39):13746-13757.

57. Chalazonitis A, et al. Bone morphogenetic proteins regulate enteric gliogenesis by modulating ErbB3 signaling. Dev Biol. 2011;350(1):64-79.

58. Chalazonitis A, et al. Bone morphogenetic protein regulation of enteric neuronal phenotypic diversity: relationship to timing of cell cycle exit. J Comp Neurol. 2008;509(5):474-492.

59. Faure C, et al. Gangliogenesis in the enteric nervous system: roles of the polysialylation of the neural cell adhesion molecule and its regulation by bone morphogenetic protein-4. Dev Dyn. 2007;236(1):44-59.

60. Fu M, et al. BMP signaling regulates murine enteric nervous system precursor migration, neurite fasciculation, and patterning via altered Ncam1 polysialic acid addition. Dev Biol. 2006;299(1):137-150.

61. Goldstein AM, et al. BMP signaling is necessary for neural crest cell migration and ganglion formation in the enteric nervous system. Mech Dev. 2005;122(6):821-833.

62. Chalazonitis A, et al. Bone morphogenetic protein-2 and -4 limit the number of enteric neurons but promote development of a TrkC-expressing neurotrophin-3-dependent subset. J Neurosci. 2004;24(17):4266-4282.

63. Southard-Smith EM, et al. Sox10 mutation disrupts neural crest development in Dom Hirschsprung mouse model. Nat Genet. 1998;18(1):60-64.

64. Lang D, et al. Pax3 is required for enteric ganglia formation and functions with Sox10 to modulate expression of c-ret. $J$ Clin Invest. 2000;106(8):963-971.

65. Lang D, Epstein JA. Sox10 and Pax3 physically interact to mediate activation of a conserved c-RET enhancer. Hum Mol Genet. 2003;12(8):937-945.

66. Betancur P, et al. Genomic code for Sox10 activation reveals a key regulatory enhancer for cranial neural crest. Proc Natl Acad Sci US A. 2010;107(8):3570-3575.

67. Lasrado R, et al. Lineage-dependent spatial and functional organization of the mammalian enteric nervous system. Science. 2017;356(6339):722-726.

68. Zechner D, et al. Bmp and Wnt/beta-catenin signals control expression of the transcription factor Olig3 and the specification of spinal cord neurons. Dev Biol. 2007;303(1):181-190.

69. Ciani L, Salinas PC. WNTs in the vertebrate nervous system: from patterning to neuronal connectivity. Nat Rev Neurosci. 2005;6(5):351-362.

70. Vivancos V, et al. Wnt activity guides facial branchiomotor neuron migration, and involves the PCP pathway and JNK and ROCK kinases. Neural Dev. 2009;4:7.

71. West KP Jr. Extent of vitamin A deficiency among preschool children and women of reproductive age. J Nutr. 2002;132(9 suppl):2857S-2866S.

72. Friedrich G, Soriano P. Promoter traps in embryonic stem cells: a genetic screen to identify and mutate developmental genes in mice. Genes Dev. 1991;5(9):1513-1523.

73. Enomoto H, et al. RET signaling is essential for migration, axonal growth and axon guidance of developing sympathetic neurons. Development. 2001;128(20):3963-3974.

74. Kilkenny C, et al. Improving bioscience research reporting: the ARRIVE guidelines for reporting animal research. Vet Clin Pathol. 2012;41(1):27-31.

75. Grant GR, et al. Comparative analysis of RNA-Seq alignment algorithms and the RNA-Seq unified mapper (RUM). Bioinformatics. 2011;27(18):2518-2528.

76. Zhou Y, et al. Metascape provides a biologist-oriented resource for the analysis of systems-level datasets. Nat Commun. 2019;10(1):1523. 